Rev. Int. Contam. Ambie. 36 (3) 623-643, 2020

https://doi.org/10.20937/RICA.53417

\title{
ESTIMACIÓN DE LA FRACCIÓN INHALADA DE CONTAMINANTES PRIMARIOS DEL AIRE EN LA CIUDAD DE MEDELLÍN
}

Estimated of intake fraction of primary air pollutants in Medellín city

\author{
Ana Catalina JARAMILLO ROJAS* y María Victoria TORO GÓMEZ
}

Grupo de Investigaciones Ambientales, Universidad Pontificia Bolivariana, Circular 1 núm. 70-01, C.P. 050031, Medellín, Colombia

*Autor para correspondencia: anacatajara@gmail.com

(Recibido: octubre 2018; aceptado: diciembre 2019)

Palabras clave: representatividad espacial, población expuesta, impacto ambiental

\section{RESUMEN}

Se realizó la estimación de la fracción inhalada de contaminantes medidos en la ciudad de Medellín, a saber, partículas suspendidas $\left(\mathrm{PM}_{10}\right.$ y $\left.\mathrm{PM}_{2.5}\right)$, monóxido de carbono $(\mathrm{CO})$ y óxidos de nitrógeno $\left(\mathrm{NO}_{\mathrm{x}}\right)$. Se empleó el método aplicable para contaminantes de tipo primario, de acuerdo con los datos de calidad del aire existentes en términos de emisiones y concentraciones, como un factor que puede utilizarse en la evaluación del impacto de las fuentes de contaminación atmosférica en la salud humana. Para obtener el cálculo se procesaron los datos de las siguientes variables: emisiones, concentración de exposición atribuible, tamaño y tasa de respiración de la población. Dada la importancia de la relación espacial y temporal de las variables, todos los datos son de 2015, último periodo en el que se encontró actualizado el inventario de emisiones atmosféricas. Se consideró el área de representatividad espacial de las estaciones de medición de calidad del aire de la ciudad de Medellín y se obtuvieron celdas con una función de similitud de concentración (CSF, por sus siglas en inglés) mayor a 0.9 en las estaciones Politécnico Jaime Isaza Cadavid (MED-PJIC) (PM10 y CO) y Universidad Nacional Núcleo el Volador (MED-UNNV) $\left(\mathrm{PM}_{2.5}\right.$ y NO $\left.\mathrm{NO}_{\mathrm{x}}\right)$. Los promedios anuales de la fracción inhalada para estas áreas de representatividad fueron de 475 y 29 ppm para $\mathrm{PM}_{10}$ y CO, respectivamente, en la estación MED-PJIC, y de 151 y 64 ppm para $\mathrm{PM}_{2.5}$ y NOx, respectivamente, en la estación MED-UNNV. Se analizaron también los valores de la fracción inhalada utilizando las tasas ponderadas de respiración por grupos de edades, así como los promedios horarios, diarios, mensuales y para cada día de la semana de los valores de concentración y emisión de contaminantes.

Key words: spatial representativeness, exposed population, environmental impact

\begin{abstract}
Intake fraction was estimated for measured pollutants in Medellín city: suspended particulates $\left(\mathrm{PM}_{10}\right.$ and $\left.\mathrm{PM}_{2.5}\right)$, carbon monoxide $(\mathrm{CO})$ and nitrogen oxides $\left(\mathrm{NO}_{\mathrm{x}}\right)$. The applicable method for primary pollutants was used, according to the existing air quality data in terms of emissions and concentrations, as a factor that can be used in the evaluation of the impact on human health due to the sources of air pollution. The data of emissions, attributable exposure concentration, amount population and
\end{abstract}


breathing rates were processed for calculation. Given the importance of the spatial and temporal relationship of the variables, all data were from 2015, the last period in which the inventory of atmospheric emissions was updated. Areas of spatial representativeness were considered, obtaining cells with a CSF greater than 0.9 in Politécnico Jaime Isaza Cadavid (MED-PJIC) ( $\mathrm{PM}_{10}$ and $\mathrm{CO}$ ) and Universidad Nacional Núcleo el Volador (MED-UNNV) $\left(\mathrm{PM}_{2.5}\right.$ and $\left.\mathrm{NO}_{\mathrm{x}}\right)$ stations. Yearly averages of the intake fraction were: 475 and $29 \mathrm{ppm}$ for $\mathrm{PM}_{10}$ and $\mathrm{CO}$, respectively, at MED-PJIC station, and 151 and $64 \mathrm{ppm}$ for $\mathrm{PM}_{2.5}$ and $\mathrm{NO}_{\mathrm{x}}$, respectively, at MED-UNNV station. The intake fraction values were analyzed too by weighted respiration rates by age groups, and by hourly, daily, monthly and daily weekday averages of concentration and pollutant emission values.

\section{INTRODUCCIÓN}

La contaminación del aire ocurre por la presencia de sustancias de diversos orígenes y composición que, en ciertas cantidades y períodos de tiempo, pueden resultar nocivas. Las más comunes por sus efectos perjudiciales para la salud y el bienestar de los humanos son las partículas menores a 10 y 2.5 $\mu \mathrm{m}\left(\mathrm{PM}_{10}\right.$ y $\mathrm{PM}_{2.5}$, respectivamente), los óxidos de azufre $\left(\mathrm{SO}_{\mathrm{x}}\right)$ y nitrógeno $\left(\mathrm{NO}_{\mathrm{x}}\right)$, el monóxido de carbono $(\mathrm{CO})$ y el ozono troposférico $\left(\mathrm{O}_{3}\right)$. Todas estas sustancias se agrupan en los denominados contaminantes criterio (IDEAM 2017a).

Estos agentes contaminantes se consideran de tipo primario, ya que son emitidos directamente a la atmósfera por una fuente, a excepción del $\mathrm{O}_{3}$ que es de tipo secundario puesto que se forma a partir de reacciones fotoquímicas en presencia de radiación solar y de precursores como $\mathrm{NO}_{\mathrm{x}}$ y los compuestos orgánicos volátiles (COV) (WHO 2006).

Según la Organización Mundial de la Salud (OMS), la carga de enfermedad (mortalidad y morbilidad) debida a la contaminación del aire se asocia con patologías como enfermedad pulmonar obstructiva crónica (EPOC), infecciones respiratorias agudas (IRA), accidentes cerebrovasculares, cardiopatías isquémicas y cáncer de pulmón, para las cuales hay suficiente evidencia epidemiológica de la relación causa-efecto (WHO 2016).

Para el caso de Colombia, el Diagnóstico Nacional de Salud Ambiental (MADS 2012) estima que las pérdidas anuales por contaminación local del aire ascienden a 5700 billones de pesos. Cerca de 5000 muertes prematuras y casi 65000 años de vida ajustados según discapacidad (AVAD) son atribuibles a esta causa. Cabe anotar que Bogotá y el Valle de Aburrá son las zonas del país en que se concentra más del $75 \%$ de la mortalidad atribuible.

La evaluación del riesgo que afronta la salud humana por fuentes de contaminación ambiental implica una evaluación de la cadena causa-efecto que vincula la emisión y el daño a la salud. Las emisiones se transportan y se transforman en el aire para generar concentraciones ambientales de contaminantes que generalmente varían en el tiempo y el espacio. Los encuentros de personas con estas concentraciones constituyen exposiciones, y su inhalación da como resultado la incorporación de los contaminantes presentes. La transferencia de estos contaminantes al interior del cuerpo genera vulnerabilidad en algunos órganos del individuo expuesto y de este modo se constituyen en dosis, variable asociada al riesgo de generación de efectos adversos para la salud (Marshall y Nazaroff 2006, Rosenbaum et al. 2007).

Para la mayoría de los contaminantes primarios, las concentraciones, las exposiciones y las dosis son proporcionales a las emisiones (Lai et al. 2000). Esta relación lineal entre emisiones y dosis sugiere que la estimación del riesgo para la salud por fuentes de contaminación ambientales puede realizarse de manera simplificada con base en la información de tres elementos: emisiones totales, fracción de admisión para una fuente distribuida (o fracción inhalada) y toxicidad basada en la ingesta (Marshall y Nazaroff 2002), donde las unidades son: masa o masa por tiempo (emisiones), masa inhalada por masa emitida (fracción inhalada) e impacto en la salud por masa inhalada (toxicidad) (Humbert et al. 2011).

En situaciones ideales se deberían conocer los tres términos; sin embargo, se puede obtener una importante visión del impacto ambiental sólo a partir de la fracción inhalada, que si bien no puede usarse específicamente como una variable independiente para estudios epidemiológicos, sí puede aportar elementos en la cuantificación del riesgo de una población y proporcionar información útil sobre las ventajas o desventajas de diferentes políticas y/o escenarios, como por ejemplo, reducción de emisiones (Oakes et al. 2014). 
La fracción inhalada refleja mediante una relación adimensional, la porción de contaminación liberada al ambiente que es tomada por una población expuesta. Además, resume cuantitativamente la relación entre la emisión y la inhalación como un solo número fácil de usar y entender. Así, una fracción inhalada de uno por millón significa que, por cada kilogramo de contaminante emitido, $1 \mathrm{mg}$ es inhalado por la población expuesta. También puede interpretarse como que es necesario reducir las emisiones en 1 $\mathrm{kg}$ si se quiere reducir en $1 \mathrm{mg}$ la cantidad inhalada (Marshall y Nazaroff 2006).

Esta fracción tomada por la población se expresa como inhalada para el caso de contaminantes presentes en el aire que ingresan en el organismo por vía respiratoria, aunque el término puede utilizarse también para contaminantes que ingresan en el organismo por ingestión o por vía dérmica (Bennett et al. 2002).

En Colombia, la norma de calidad del aire o nivel de inmisión busca garantizar un ambiente sano y minimizar el riesgo para la salud humana que pueda causar la exposición a contaminantes de la atmósfera (MADS 2017). Para tal efecto se establecen los niveles máximos permisibles para los contaminantes criterio $\mathrm{SO}_{2}, \mathrm{CO}, \mathrm{NO}_{2}, \mathrm{O}_{3}$ y partículas $\mathrm{PM}_{10}$ y $\mathrm{PM}_{2.5}$, los cuales son de obligatoria medición por parte de las autoridades ambientales en las áreas de su jurisdicción.

El Valle de Aburrá está integrado por 10 municipios: Barbosa, Girardota, Copacabana, Bello, Medellín, Envigado, Sabaneta, Itagüí, La Estrella y Caldas; tiene una extensión de $1152 \mathrm{~km}^{2}$, de los cuales 340 $\mathrm{km}^{2}$ corresponden a área urbana y $812 \mathrm{~km}^{2}$ a área rural. Según los resultados del censo poblacional realizado en 2005, la población total era de 3306 490 habitantes distribuidos de la siguiente forma: $94.53 \%$ en área urbana y $5.47 \%$ en área rural. La mayoría de la población urbana y rural está localizada en Medellín (67\%), seguida por los municipios de Bello, Itagüí y Envigado (AMVA 2007).

Los resultados de operación de la red de medición de calidad del aire, meteorología y ruido de esta región indican que existe una problemática de contaminación del aire, considerándose como el caso más crítico el de las partículas de tamaño $\mathrm{PM}_{2.5}$, ya que en todas las estaciones que registran mediciones en el Valle de Aburrá se han alcanzado indicadores de calidad de aire "dañino para la salud" asociados a este contaminante. Estos indicadores han llevado a la autoridad ambiental a declarar niveles de contingencia atmosférica en 2016 (AMVA 2016), alerta roja fase I en 2017 (IDEAM 2017b) y nivel de alerta en 2018 (AMVA 2018).
No obstante, estos valores e índices en términos de concentración (calidad del aire) no son el mejor indicador para relacionar las emisiones y los efectos. La cantidad inhalada por las personas, la asociación con la concentración en el aire o la cantidad emitida de un contaminante pueden ser indicadores más adecuados de los posibles impactos adversos para la salud (Marshall y Nazaroff 2006, UNEP 2016).

La estimación de la fracción inhalada puede realizarse para una o múltiples fuentes de emisión, así como para uno o varios contaminantes. En la literatura se encuentran diversos valores de fracción inhalada, incluyendo contaminantes primarios y secundarios, así como fuentes de emisión variadas. En el caso de valores de fracción inhalada de contaminantes de tipo primario aplicados para áreas urbanas, existen varios estudios de referencia (Cuadro I), entre los cuales destaca el de Apte et al. (2012), en el cual se calculó la fracción inhalada intraurbana de las emisiones de contaminantes primarios distribuidos a nivel del suelo en todas las ciudades del mundo con una población de 100000 en el año 2000. De un total de 3646 ciudades, se analizaron 26 ciudades colombianas, entre ellas Medellín. El valor de la fracción inhalada intraurbana fue de $220 \mathrm{ppm}$, que es el segundo valor más alto, por debajo de Daca (Bangladés), con un valor de $260 \mathrm{ppm}$. En el modelo empleado se consideraron como variables de entrada los datos meteorológicos de velocidad del viento y altura de mezcla, la frecuencia respiratoria y los datos de población, área terrestre y localización (latitud y longitud) de cada área urbana. Respecto al perfil de emisiones, se asumió que la fracción inhalada es independiente de las tasas de emisión, ya que las concentraciones de contaminantes primarios escalan linealmente con las emisiones.

En este estudio se utilizaron los datos de concentración ambiental y de emisión (provenientes de fuentes fijas y móviles) de los contaminantes primarios $\mathrm{PM}_{10}, \mathrm{PM}_{2.5}, \mathrm{NO}_{\mathrm{x}}$ y $\mathrm{CO}$ evaluados en la ciudad de Medellín como municipio más representativo del Valle de Aburrá en términos de área y población, para estimar la fracción inhalada, factor que puede ser utilizado en la evaluación del impacto en la salud humana por fuentes de contaminación atmosférica.

\section{MATERIALES Y MÉTODOS}

Al igual que las emisiones y concentraciones, la fracción inhalada puede determinarse a través de diferentes métodos. Se ha seleccionado el método de estimación de fracción inhalada aplicable para 
CUADRO I. VALORES DE LA FRACCIÓN INHALADA PARA CONTAMINANTES DE TIPO PRIMARIO OBTENIDOS EN OTROS ESTUDIOS

\begin{tabular}{|c|c|c|c|}
\hline Referencia & Ubicación & Contaminante & $\begin{array}{l}\text { Valor promedio de la fracción inhalada } \\
\qquad(\mathrm{ppm})\end{array}$ \\
\hline Marshall et al. (2003) & California & $\mathrm{CO}$ & $\begin{array}{l}32-46 \\
\text { (anual) }\end{array}$ \\
\hline \multirow[t]{3}{*}{ Stevens et al. (2007) } & \multirow[t]{3}{*}{$\begin{array}{l}\text { Ciudad de } \\
\text { México }\end{array}$} & Contaminantes primarios & $\begin{array}{l}40 \text { (anual) } \\
120 \text { (anual) }\end{array}$ \\
\hline & & $\mathrm{PM}_{2.5}$ primario & $\begin{array}{l}26 \text { (anual) } \\
120 \text { (anual) }\end{array}$ \\
\hline & & $\mathrm{CO}$ & $\begin{array}{l}61(\text { mensual) } \\
120 \text { (mensual) }\end{array}$ \\
\hline Luo et al. (2010) & Hong Kong & $\mathrm{CO}$ & $\begin{array}{c}200-300 \\
\text { (anual) }\end{array}$ \\
\hline \multirow[t]{2}{*}{ Apte et al. (2012) } & Medellín & \multirow{2}{*}{$\begin{array}{l}\text { Contaminantes } \\
\text { primarios }\end{array}$} & 220 (anual) \\
\hline & Daca & & 260(anual) \\
\hline \multirow[t]{2}{*}{ Du et al. (2012) } & \multirow[t]{2}{*}{ Beijing } & $\mathrm{PM}_{2.5}$ & $153 \pm 97$ (diario-24h) \\
\hline & & $\mathrm{NO}_{\mathrm{x}}$ & $70 \pm 32$ (diario-24h) \\
\hline \multirow[t]{3}{*}{ Tainio et al. (2014) } & \multirow[t]{3}{*}{ Varsovia } & $\mathrm{PM}_{10}$ & 51 (anual) \\
\hline & & $\mathrm{PM}_{2.5}$ & 50 (anual) \\
\hline & & $\mathrm{NO}_{\mathrm{x}}$ & 13 (anual) \\
\hline \multirow[t]{4}{*}{ Holnicki et al. (2018) } & \multirow{4}{*}{$\begin{array}{l}\text { Varsovia } \\
\text { (sólo fuentes } \\
\text { móviles) }\end{array}$} & $\mathrm{PM}_{10}$ & 37 (anual) \\
\hline & & $\mathrm{PM}_{2.5}$ & 39 (anual) \\
\hline & & $\mathrm{NO}_{\mathrm{x}}$ & 24 (anual) \\
\hline & & $\mathrm{CO}$ & 36 (anual) \\
\hline
\end{tabular}

$\mathrm{CO}$ : monóxido de carbono, $\mathrm{PM}_{2.5}$ : partículas menores a $2.5 \mu \mathrm{m}, \mathrm{NO}_{\mathrm{x}}$ : óxidos de nitrógeno, $\mathrm{PM}_{10}$ : partículas menores a $10 \mu \mathrm{m}$

contaminantes de tipo primario, en concordancia con los datos de calidad del aire existentes para la ciudad de Medellín según emisiones y concentraciones.

La fracción de contaminación liberada al ambiente que es tomada por una población expuesta se puede calcular mediante la ecuación 1 como:

Fracción inhalada $(i F)=\frac{\text { Población que inhala }}{\text { Total emisiones }}$

$$
i F=\frac{\int_{T_{1}}^{\infty}\left(\sum_{i=1}^{P_{1}}\left(C_{i}(t) Q_{i}(t)\right)\right) d t}{\int_{T_{1}}^{T_{2}} E(t) d t}
$$

donde $T_{1}$ y $T_{2}$ son los tiempos de inicio y finalización de las emisiones, $P$ es el número de personas en la población expuesta, $Q_{\mathrm{i}}(t)$ es la tasa de respiración para un individuo $i$ en el tiempo $t, C i(t)$ es la concentración incremental atribuible a una fuente específica en el tiempo $t$ en la zona de respiración del individuo $i$ y $E(t)$ son las emisiones de la fuente en el tiempo $t$ (Marshall et al. 2003).

Dado que las cuatro variables definidas en la ecuación 1 tienen alta dependencia espacial y temporal, se ha considerado como criterio de ubicación el área de representatividad espacial de las estaciones de medición de calidad del aire que suministran los datos de concentración ambiental de cada uno de los contaminantes definidos. Como criterio de temporalidad, el último inventario de emisiones atmosféricas del Valle de Aburrá disponible para la fecha del estudio era de 2015 (AMVA-UPB 2017a), así que los datos de las variables corresponden a este mismo periodo de tiempo. 


\section{Estaciones de medición de calidad del aire}

La red de medición de calidad de aire del Valle de Aburrá tuvo 31 estaciones (entre fijas y móviles) a diciembre de 2017, distribuidas en los diferentes municipios que forman parte de la jurisdicción del Área Metropolitana del Valle de Aburrá (AMVA-EAFIT 2017). De éstas, 14 estuvieron ubicadas en la ciudad de Medellín y cinco midieron durante el 2015 por lo menos uno de los contaminantes primarios, excepto $\mathrm{SO}_{\mathrm{x}}$, los cuales no fueron evaluados en ninguna de las estaciones de la ciudad. La medición fue realizada con equipos de tipo automático, con análisis de la muestra de forma continua, utilizando principalmente métodos ópticos y electrónicos con transmisión de datos en tiempo real (MAVDT 2008), lo cual garantizó el acceso a valores de concentración horarios de cada contaminante. En el cuadro II se presenta la distribución de los contaminantes medidos en cada una de las estaciones y en la figura 1 el mapa (1:40 000) de su distribución espacial para el área urbana del municipio de Medellín.

\section{CUADRO II. CONTAMINANTES PRIMARIOS EVALUA- DOS EN LA CIUDAD DE MEDELLÍN EN 2015}

\begin{tabular}{lcccc}
\hline Nombre de la estación & \multicolumn{4}{c}{ Contaminantes } \\
\cline { 2 - 5 } & $\mathrm{PM}_{10}$ & $\mathrm{PM}_{2.5}$ & $\mathrm{NO}_{\mathrm{x}}$ & $\mathrm{CO}$ \\
\hline Universidad Nacional & & & & \\
Núcleo el Volador & $\mathrm{X}$ & $\mathrm{X}$ & $\mathrm{X}$ & \\
Museo de Antioquia & $\mathrm{X}$ & & $\mathrm{X}$ & $\mathrm{X}$ \\
Politécnico Jaime Isaza Cadavid & & $\mathrm{X}$ & & \\
Tanques LAYE & $\mathrm{X}$ & & & \\
Éxito San Antonio & & & & \\
\hline
\end{tabular}

$\mathrm{PM}_{10}$ : partículas menores a $10 \mu \mathrm{m}, \mathrm{PM}_{2.5}$ : partículas menores a $2.5 \mu \mathrm{m}, \mathrm{NO}_{\mathrm{x}}$ : óxidos de nitrógeno, $\mathrm{CO}$ : monóxido de carbono

\section{Representatividad espacial de las estaciones de medición}

Una medición puntual se considera representativa del promedio de un área mayor si la probabilidad de que la diferencia al cuadrado entre la medición del punto y del área es menor que un criterio umbral en más del $90 \%$ del tiempo (Nappo et al. 1982). Este método se basa en el análisis de series temporales de concentraciones modelizadas por cuadrículas, extraídas de estaciones de medición y de sus alrededores mediante una función de similitud de concentración (CSF, por sus siglas en inglés). Cabe resaltar que todas las celdas en que se verifica una función de frecuencia mayor a 0.9 forman parte del área de representatividad del sitio de interés (Piersanti et al. 2015).

En la aplicación de este método se conservó la resolución espacial de celdas de $1 \mathrm{~km}^{2}$ definida en el modelo de emisiones atmosféricas del área metropolitana del Valle de Aburrá (MODEAM). Se identificaron las celdas en las que se encuentran ubicadas las estaciones de medición de calidad del aire ya definidas y se compararon, para cada contaminante, los datos de concentración del punto donde se encuentra ubicada la estación con el valor promedio de cada una de las celdas (tanto en la celda que se encontraba ubicada la estación como en las celdas contiguas).

Ya que sólo se dispuso de valores de concentración medidos en el punto donde se encuentra ubicada cada estación de medición de calidad del aire, los datos fueron obtenidos de los pronósticos por localización disponibles en el sistema de información metropolitano de la calidad del aire (SIMECA), permitiendo visualizar el valor de concentración de cada contaminante en cualquier punto del Valle de Aburrá (AMVA-UPB 2018a) a partir de simulaciones realizadas con el modelo de calidad del aire comprehensivo con extensiones (CAMx, por sus siglas en inglés). Éste es un modelo de dispersión fotoquímico eureliano en el que los valores de concentración de cada especie química son el resultado de la simulación de valores de emisiones, dispersión, reacción química y remoción de contaminantes (AMVA-UPB 2017b). El periodo de análisis comprendió del 1 al 31 de enero de 2018, garantizando la existencia de valores tanto en la estación de medición como en el modelo de pronóstico para evaluar el desempeño de los datos utilizados. El valor umbral empleado fue del $25 \%$ en concordancia con el criterio de representatividad temporal definido en el Manual de Operación de Sistemas de Vigilancia de la Calidad del Aire (MAVDT 2008).

\section{Variables de cálculo para la fracción inhalada Emisiones}

Con la aplicación del MODEAM (AMVA-UPB 2018b) se realizó la estimación de las emisiones en el área metropolitana del Valle de Aburrá, haciendo la distinción en su origen entre fuentes fijas, fuentes móviles y fuentes de área de los contaminantes $\mathrm{CO}$, $\mathrm{NO}_{\mathrm{x}}, \mathrm{SO}_{\mathrm{x}}, \mathrm{COV}$ y partículas $\left(\mathrm{PM}_{10}\right.$ y $\left.\mathrm{PM}_{2.5}\right)$, con una resolución espacial en celdas de $1 \mathrm{~km}^{2}$. De estos contaminantes se excluyeron del análisis los COV y $\mathrm{SO}_{\mathrm{x}}$, ya que no fueron evaluados en las estaciones de calidad del aire. 


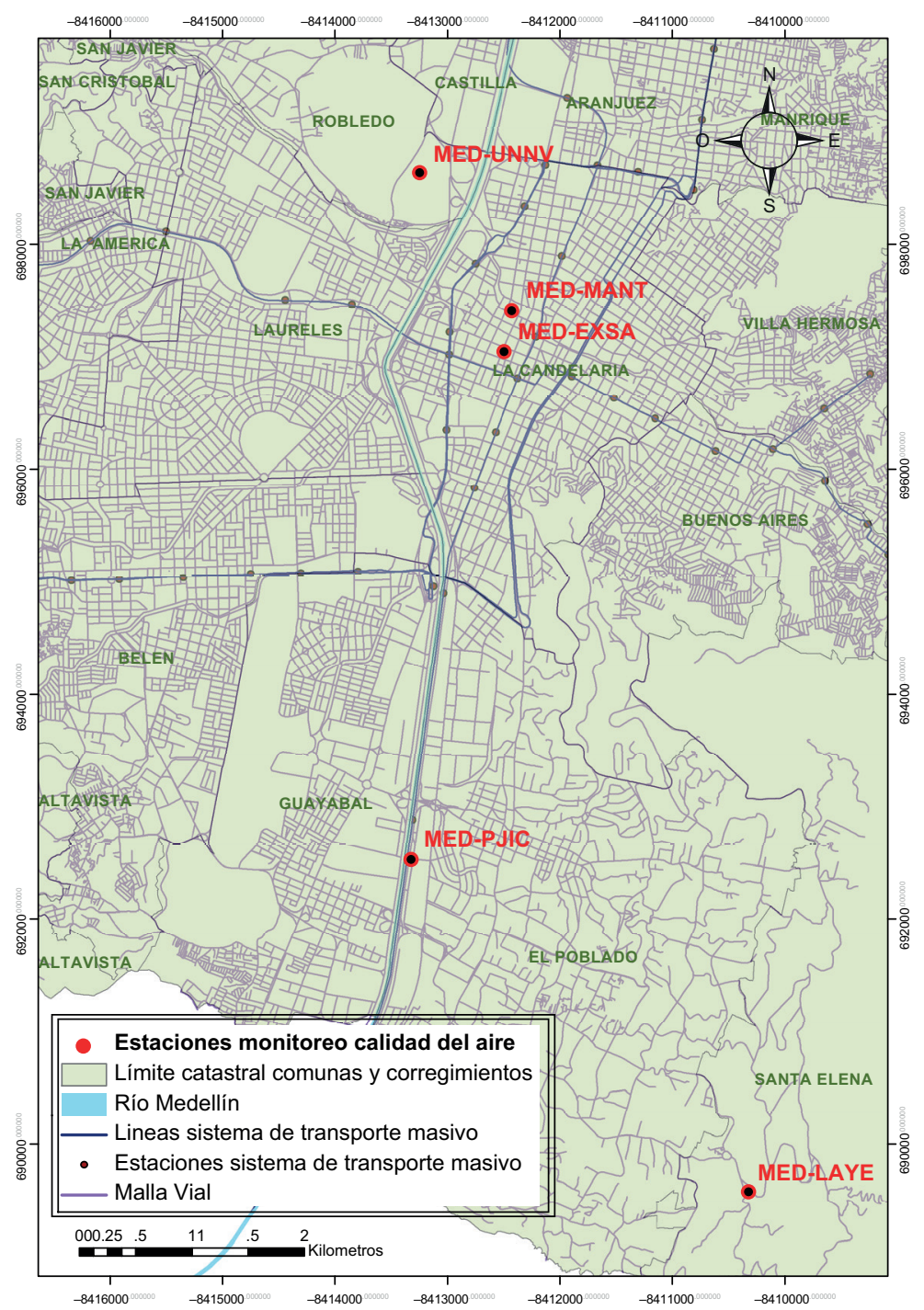

Fig. 1. Ubicación de las estaciones de medición de calidad del aire que evaluaron contaminantes primarios durante 2015. MED-UNNV: Universidad Nacional Núcleo el Volador; MED-MANT: Museo de Antioquia; MEDEXSA: Éxito San Antonio; MED-PJIC: Politécnico Jaime Isaza Cadavid; MED-LAYE: Tanques LAYE

Las emisiones de contaminantes a partir de fuentes móviles estuvieron disponibles como promedios de emisión diaria $(\mathrm{kg} /$ día $)$ y horaria $(\mathrm{kg} / \mathrm{h})$. Las correspondientes a fuentes fijas se estimaron en escala anual $(\mathrm{Mg} / \mathrm{año})$ y se llevaron a emisiones en escalas diaria y horaria considerando los tiempos de operación de los equipos asociados con estas emisiones. Las emisiones generadas por fuentes de área no fueron consideradas para el análisis ya que el inventario para este tipo de fuente incluyó emisiones de COV, contaminantes no incluidos en el estudio. Para éste se consideraron las emisiones totales (provenientes de fuentes fijas y móviles) de las celdas que tuvieron correspondencia con el área de representatividad espacial de cada una de las estaciones de medición.

\section{Concentración de exposición atribuible}

Con el uso de la herramienta en línea de seguimiento de la calidad del aire en el Valle de Aburrá (SCALAR), que posibilita la consulta y análisis del comportamiento de los contaminantes medidos en las estaciones de registro de calidad del aire del Área Metropolitana del Valle de Aburrá a partir de datos validados por el operador de la red (AMVA-UPB 
2018c), se obtuvieron las cifras de concentración horaria de los contaminantes en aquellas estaciones, pudiendo determinarse un área de representatividad espacial equivalente mínimo a una de las celdas de $1 \mathrm{~km}^{2}$ definidas en el MODEAM.

\section{Tamaño de población}

La Alcaldía de Medellín realizó en conjunto con el Departamento Administrativo Nacional de Estadísticas (DANE) las proyecciones de población residente de las 16 comunas y de los cinco corregimientos del municipio de Medellín de acuerdo con el género y grupos de edad quinquenales para el período 20062015, a partir del censo general 2005 (Municipio de Medellín-DANE 2011). Por otro lado, a partir del Plan de Ordenamiento Territorial (POT) y del Plan de Desarrollo Municipal fue posible acceder a la información relacionada con la distribución geográfica del territorio por comunas y corregimientos (áreas). Para cada una de las áreas de representatividad espacial de las estaciones de medición se calcularon, a partir de los datos poblacionales de la comuna o comunas correspondientes, la densidad poblacional (personas/ $\mathrm{km}^{2}$ ). Esta cifra se multiplicó por el tamaño del área de representatividad espacial de cada estación $\left(\mathrm{km}^{2}\right)$ para estimar la población de cada área de estudio.

\section{Tasa de respiración}

La Agencia de Protección Ambiental de Estados Unidos cuenta con un manual de factores de exposición que contiene resúmenes de estudios científicamente fundamentados para derivar recomendaciones sobre factores de exposición (USEPA 2011). Este manual proporciona tasas de respiración (o inhalación) a corto y a largo plazo, por grupos de edad, género y nivel de actividad. Acorde con la distribución poblacional de la comuna o comunas que correspondieron con el área de representatividad espacial de cada estación de medición, disponible por género y grupos de edad quinquenales, se utilizaron como referencia los valores promedio de tasa de respiración por género y grupo de edad para obtener el promedio ponderado de la tasa de respiración aplicable a cada área de estudio.

\section{RESULTADOS Y DISCUSIÓN}

\section{Representatividad espacial de las estaciones de medición}

Se aplicó la función de similitud de concentración (CSF, por sus siglas en inglés) usando los datos promedio diarios de concentración en cada una de las es- taciones de medición definidas y sus celdas contiguas. Se conservó la resolución espacial de celdas de $1 \mathrm{~km}^{2}$ y fue posible definir las áreas de representatividad espacial de las estaciones de medición y contaminantes de la ciudad de Medellín: Politécnico Jaime Isaza Cadavid (MED-PJIC) $\left(\mathrm{PM}_{10}\right.$ y CO), y Universidad Nacional Núcleo el Volador (MED-UNNV) $\left(\mathrm{PM}_{2.5}\right.$ y $\mathrm{NO}_{\mathrm{x}}$ ). Además, se obtuvieron celdas contiguas con una CSF mayor a 0.9 (Fig. 2).

El área de representatividad espacial para los contaminantes $\mathrm{PM}_{10}$ y CO en la estación MED-PJIC correspondió a dos celdas en dirección suroriente de la ubicación de la estación de medición, con un área de $2.04 \mathrm{~km}^{2}$. Para los contaminantes $\mathrm{PM}_{2.5}$ y NO en la estación MED-UNNV, el área de representatividad espacial correspondió a tres celdas en dirección norte de la ubicación de la estación, con un área de $3.06 \mathrm{~km}^{2}$.

Para las demás estaciones de medición y contaminantes, los valores de CSF fueron menores a 0.9, por lo que las áreas de representatividad en estos casos pudieron ser menores a $1 \mathrm{~km}^{2}$ y/o no coincidir con la distribución espacial de las celdas del modelo de emisiones, ya que la mayoría quedaron ubicadas cerca de uno de los vértices de la celda (Fig. 3).

Al realizar el análisis de representatividad espacial, sobresalieron los resultados de las estaciones de medición Museo de Antioquia (MED-MANT) y Éxito San Antonio (MED-EXSA) para el contaminante $\mathrm{PM}_{10}$, puesto que los valores de CSF son iguales. Estas dos estaciones estuvieron ubicadas espacialmente en la misma celda, separadas entre sí por una distancia lineal de $370 \mathrm{~m}$. Los valores de concentración pronosticados en el SIMECA para estas localizaciones fueron los mismos. Al comparar los datos de las mediciones de ambas estaciones se obtuvo una diferencia promedio del $23 \%$. En este orden de ideas sólo sería necesaria una de las dos estaciones de medición en esta área, ya que existió alta similitud entre los resultados obtenidos.

Otras áreas de representatividad espacial evaluadas con el mismo método de CSF en Italia, reportaron valores que inician con áreas de $0.067 \mathrm{~km}^{2}$ para una estación de medición industrial de $\mathrm{PM}_{10}$ (Vitali et al. 2016), hasta rangos de 220 a $4500 \mathrm{~km}^{2}$ para estaciones de medición de fondo rural de $\mathrm{PM}_{2.5} \mathrm{y}$ $\mathrm{O}_{3}$ (Piersanti et al. 2015). El análisis de áreas de representatividad para sitios urbanos de unos pocos kilómetros cuadrados obligó a contar con datos de emisión por cuadrículas de una resolución menor a $4 \mathrm{~km}^{2}$ (Righini et al. 2014).

La Directiva 2008/50/CE (CE 2008) aplicable para los países de la Unión Europea recomienda la 

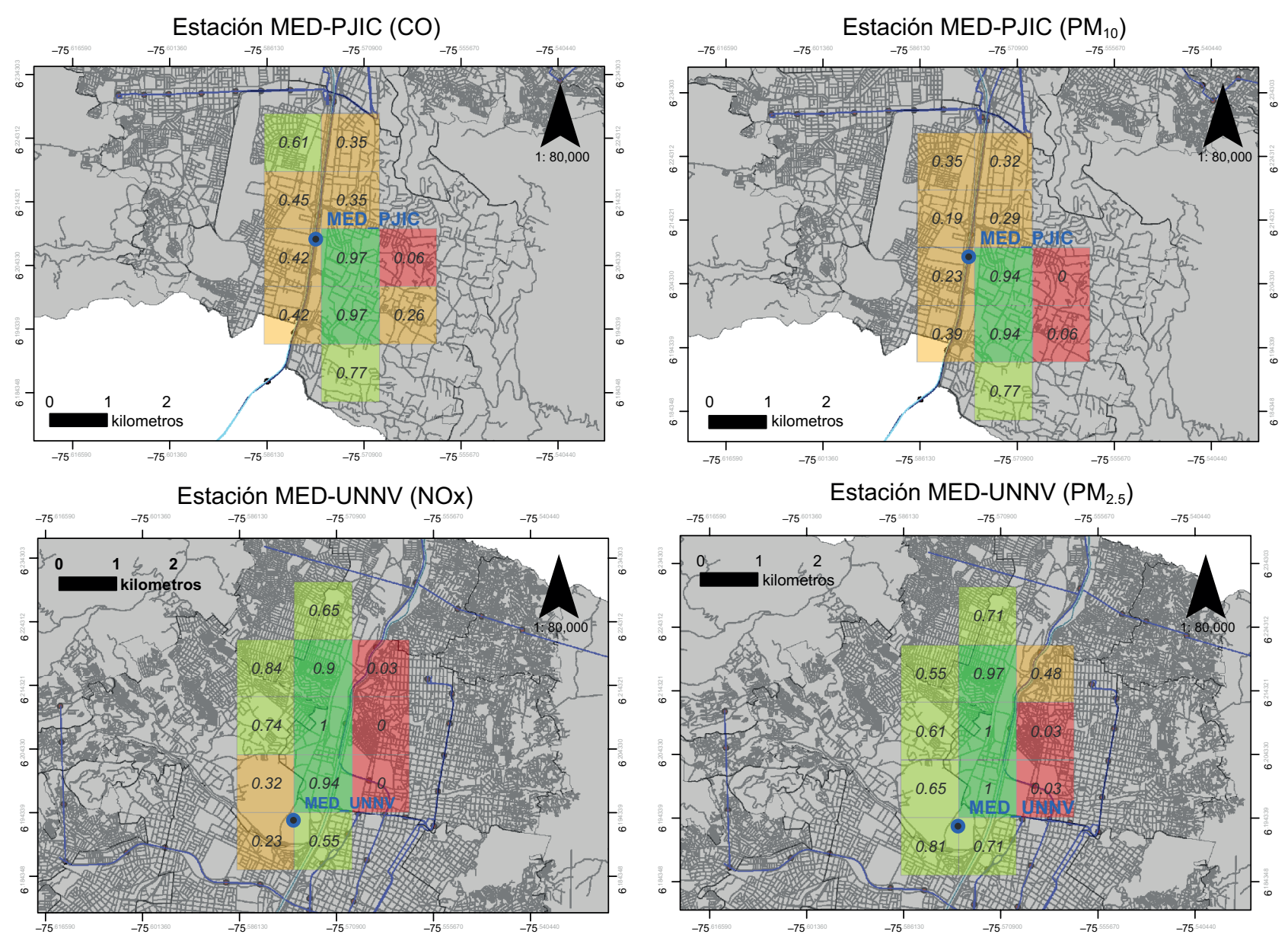

\begin{tabular}{|c|c|c|c|c|}
\hline \multirow[t]{3}{*}{ • } & \multirow{3}{*}{$\begin{array}{l}\text { Estación monitoreo calidad del aire } \\
\text { Límite catastral comunas y corregimientos } \\
\text { Río Medellín }\end{array}$} & \multirow{3}{*}{$\begin{array}{l}\text { Lineas sistema de transporte masivo } \\
\text { - Estaciones sistema de transporte masivo } \\
\text { Malla Vial }\end{array}$} & \multicolumn{2}{|l|}{ CSF por celda } \\
\hline & & & $0.00-0.09$ & $0.50-0.89$ \\
\hline & & & $0.10-0.49$ & $0.90-1.00$ \\
\hline
\end{tabular}

Fig. 2. Área de representatividad espacial con resultados de función de similitud de concentración (CSF, por sus siglas en inglés) mayores a 0.9 para $\mathrm{CO}$ y partículas menores a $10 \mu \mathrm{m}\left(\mathrm{PM}_{10}\right)$ en la estación Politécnico Jaime Isaza Cadavid (MED-PJIC); y NO y partículas menores a $2.5 \mu \mathrm{m}\left(\mathrm{PM}_{2.5}\right)$ en la estación Universidad Nacional Núcleo el Volador (MED-UNNV)

ubicación de puntos de medición que sean representativos de un segmento de calle no inferior a $100 \mathrm{~m}$ de longitud en los emplazamientos de tráfico y de al menos $250 \times 250 \mathrm{~m}$ en los emplazamientos industriales.

Para Colombia, como parte de los parámetros de diseño de los sistemas de vigilancia de calidad del aire (SVCA), existen criterios de macro y micro localización de puntos de muestreo, que incluyen consideraciones según tipo de contaminante, objetivo de la medición, condiciones de seguridad y logística, exposición de los tomadores de muestras y sensores, aspectos visuales y arquitectónicos, así como distancias a fuentes menores y a vías (MAVDT 2010).

\section{Incertidumbre en los datos de concentración}

Los datos de concentración empleados para el cálculo de la CSF en la determinación de las áreas de representatividad espacial de las estaciones de medición fueron valores pronosticados a partir del CAMx. Al ser obtenidos por modelización, tuvieron asociada una incertidumbre según el ajuste entre la realidad y los resultados obtenidos como aplicación del modelo. La forma más utilizada para lograr una 

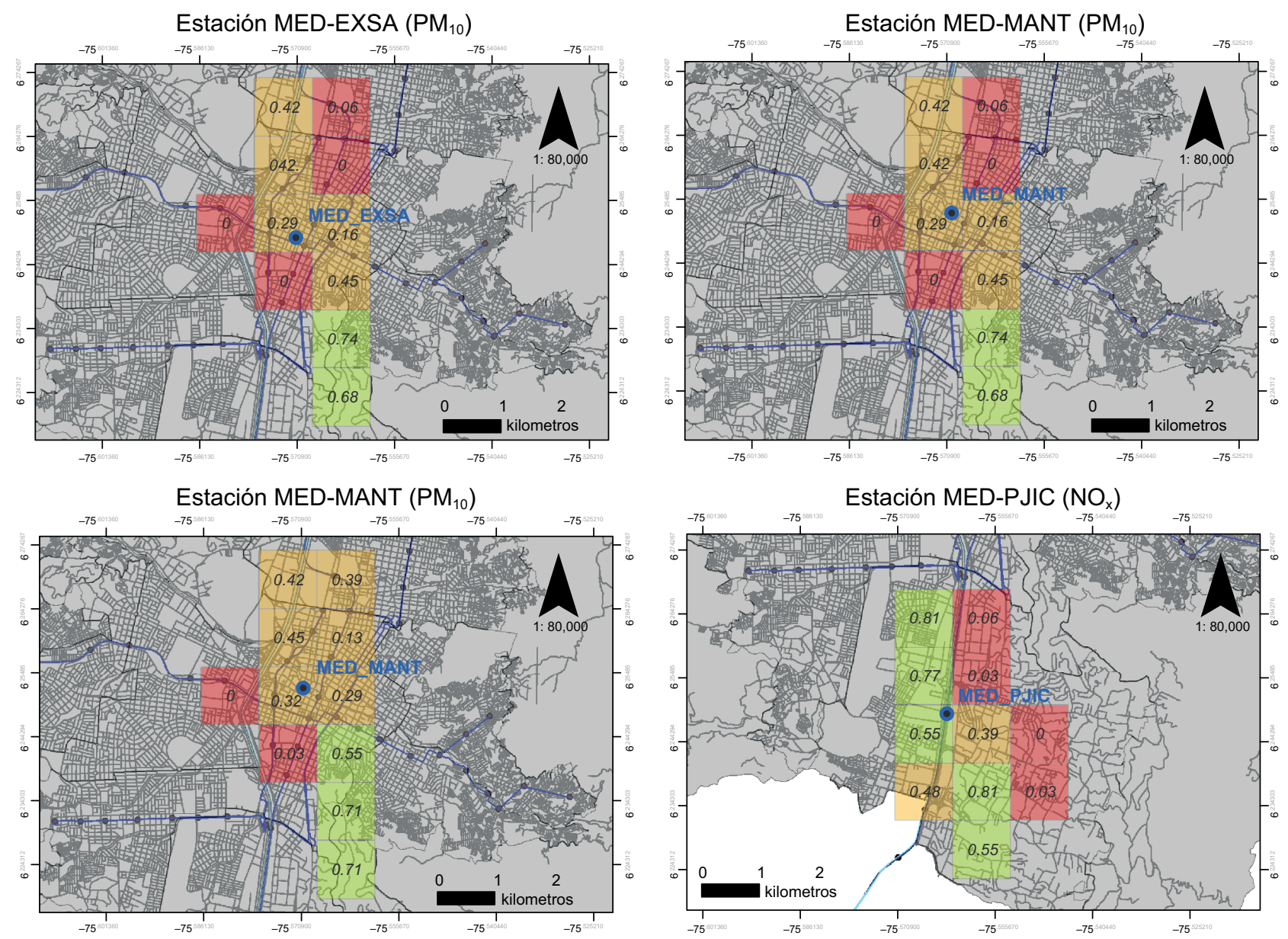

Estación MED-MANT $\left(\mathrm{PM}_{2.5}\right)$
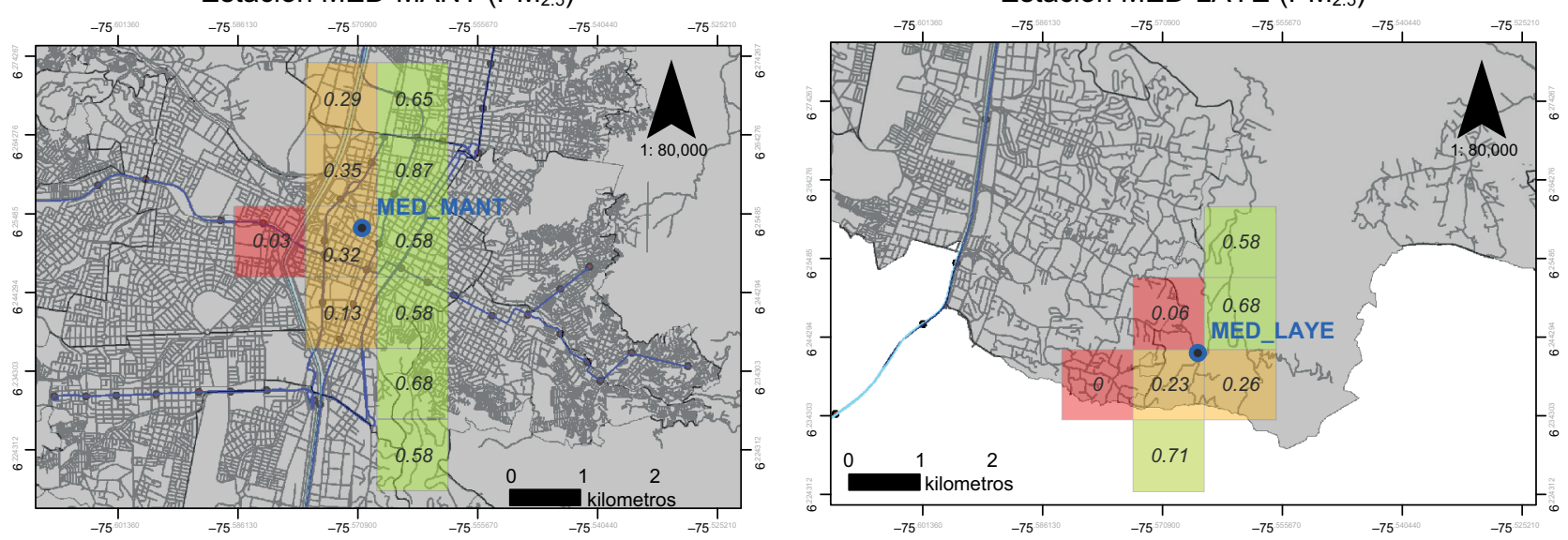

\begin{tabular}{|c|c|c|c|c|}
\hline \multirow[t]{2}{*}{$\bullet$} & \multirow{2}{*}{$\begin{array}{l}\text { Estación monitoreo calidad del aire } \\
\text { Límite catastral comunas y corregimientos }\end{array}$} & \multirow{2}{*}{$\begin{array}{l}\text { Líneas sistema de transporte masivo } \\
\text { - Estaciones sistema de transporte masivo }\end{array}$} & \multicolumn{2}{|l|}{ CSF por celda } \\
\hline & & & $0.00-0.09$ & $0.50-0.89$ \\
\hline & Río Medellín & — Malla Vial & $0.10-0.49$ & $0.90-1.00$ \\
\hline
\end{tabular}

Fig. 3. Área de representatividad espacial con resultados de función de similitud de concentración (CSF, por sus siglas en inglés) menores a 0.9 para partículas menores a $10 \mu \mathrm{m}\left(\mathrm{PM}_{10}\right)$ en las estaciones Exito San Antonio (MED-EXSA) y Museo de Antioquia (MED-MANT); óxidos de nitrógeno $\left(\mathrm{NO}_{\mathrm{x}}\right)$ en las estaciones Museo de Antioquia (MED-MANT) y Politécnico Jaime Isaza Cadavid (MED-PJIC); y partículas menores a $2.5 \mu \mathrm{m}\left(\mathrm{PM}_{2.5}\right)$ en las estaciones Museo de Antioquia (MED-MANT) y Tanques LAYE (MED-LAYE) 
medida de simulación del mundo real por medio de un modelo de calidad del aire es comparando los resultados de salida del modelo con un conjunto de mediciones directas (IDEAM 2002).

De esta forma, para evaluar el desempeño del modelo empleado (incertidumbre) se compararon los promedios diarios de concentración de los datos de pronóstico obtenidos del SIMECA con los de las mediciones de las estaciones de calidad del aire. Se calculó entonces un porcentaje de desviación entre los datos medidos (reales) y los datos pronosticados (con el modelo CAMx). Se presenta la gráfica de nube de puntos con tres líneas auxiliares 1:1, 2:1 y $1: 2$, donde $1: 1$ representa una igualdad entre los datos medidos y pronosticados, 2:1 indica que los datos pronosticados por el modelo están sobreestimados y 1:2, que el modelo subestima los valores de concentración.

El CO evaluado en la estación MED-PJIC fue el contaminante con mejor desempeño ( $71.5 \%$ de rendimiento). La mayoría de los puntos se ubicaron entre las líneas de tendencia auxiliares $2: 1$ y $1: 2$, quedando muy pocos puntos fuera de ellas (Fig. 4). Este contaminante y el ozono $\left(\mathrm{O}_{3}\right)$ son utilizados en el modelo CAMx como validadores, ya que permiten ajustar la dispersión vertical que en los modelos de calidad del aire tiene un impacto directo en las concentraciones superficiales simuladas (Foy et al. 2007, Jeričević y Večenaj 2009). Por esta razón, el

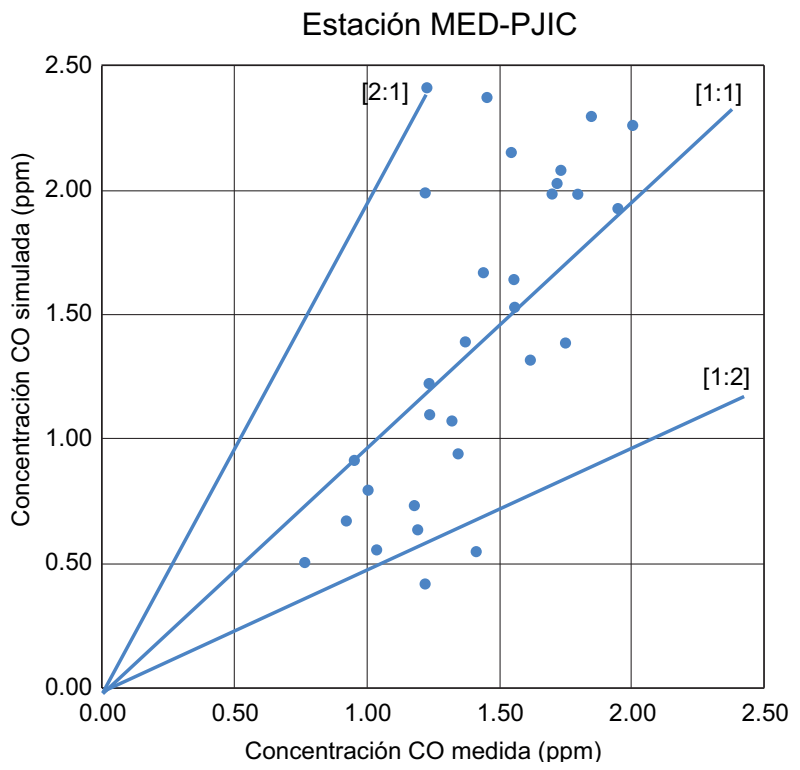

Fig. 4. Comparación de datos de medición vs simulados con el modelo de calidad del aire comprehensivo con extensiones (CAMx, por sus siglas en inglés) para el $\mathrm{CO}$ en la estación Politécnico Jaime Isaza Cadavid (MED-PJIC)
CO fue uno de los contaminantes con los que se obtuvieron mejores resultados de rendimiento durante la aplicación del modelo.

En el caso del $\mathrm{PM}_{2.5}$, el modelo tendió a subestimar sus concentraciones en todas las estaciones evaluadas (Fig. 5). Los pronósticos más ajustados correspondieron a la estación MED-UNNV (52.5\%). Para la estación MED-MANT el desempeño del modelo fue del $26.5 \%$, debiendo ajustarse los factores de emisión para mejorar el inventario por fuentes móviles en esta área (tipo de fuente más significativa). En la estación Tanques LAYE (MED-LAYE) se dispuso de poca información sobre el inventario de emisiones de esta zona, por lo que el desempeño del modelo fue del $22.8 \%$.

Las concentraciones de $\mathrm{PM}_{10}$ fueron subestimadas en todos los casos por el modelo (Fig. 6). Este contaminante pertenece al tipo de partículas gruesas que se asocian en el modelo CAMx con las especies de material particulado primario de corteza gruesa (CCRS, por sus siglas en inglés) y otros primarios gruesos (CPRM, por sus siglas en inglés). El rendimiento del modelo fue bajo, ya que el proceso químico en aerosoles está enfocado a partículas finas (RE 2016). El mejor de los resultados fue del 10.6\% para la estación MED-PJIC, seguido de $3 \%$ y $2.8 \%$ para las estaciones MED-EXSA y MED-MANT, respectivamente.

Respecto del contaminante $\mathrm{NO}_{\mathrm{x}}$, en la estación MED-MANT el modelo presentó un buen desempeño (41 \%) con algunos de los valores subestimados. Para las estaciones MED-UNNV y MED-PJIC el modelo tendió a sobreestimar los valores de concentración, manifestando un mejor desempeño en la estación MED-UNNV con un $17.7 \%$ (Fig. 7). En el modelo CAMx, el $\mathrm{NO}_{\mathrm{x}}$ es altamente reactivo e incluye las especies primarias de óxido nítrico $(\mathrm{NO})$ y dióxido de nitrógeno $\left(\mathrm{NO}_{2}\right)$.

\section{Densidad poblacional y tasas de respiración}

En la estación MED-PJIC, el área de representatividad espacial se ubicó en la comuna de El Poblado, que posee un área de $14.74 \mathrm{~km}^{2}$, una población total de 128839 habitantes y una densidad poblacional de 8741 personas $/ \mathrm{km}^{2}$. La tasa de respiración ponderada global de acuerdo con los grupos de edad por género fue de $16.40 \mathrm{~m}^{3} /$ día.

Para el caso de la estación MED-UNNV, el área de representatividad espacial se distribuyó en cinco comunas: Castilla (74.5\%), Aranjuez (12.1\%), Robledo (10.2\%), 12 de Octubre (2.5\%) y La Candelaria $(0.8 \%)$. Se calculó una densidad poblacional ponderada de 25158 personas $/ \mathrm{km}^{2}$ acorde con el 

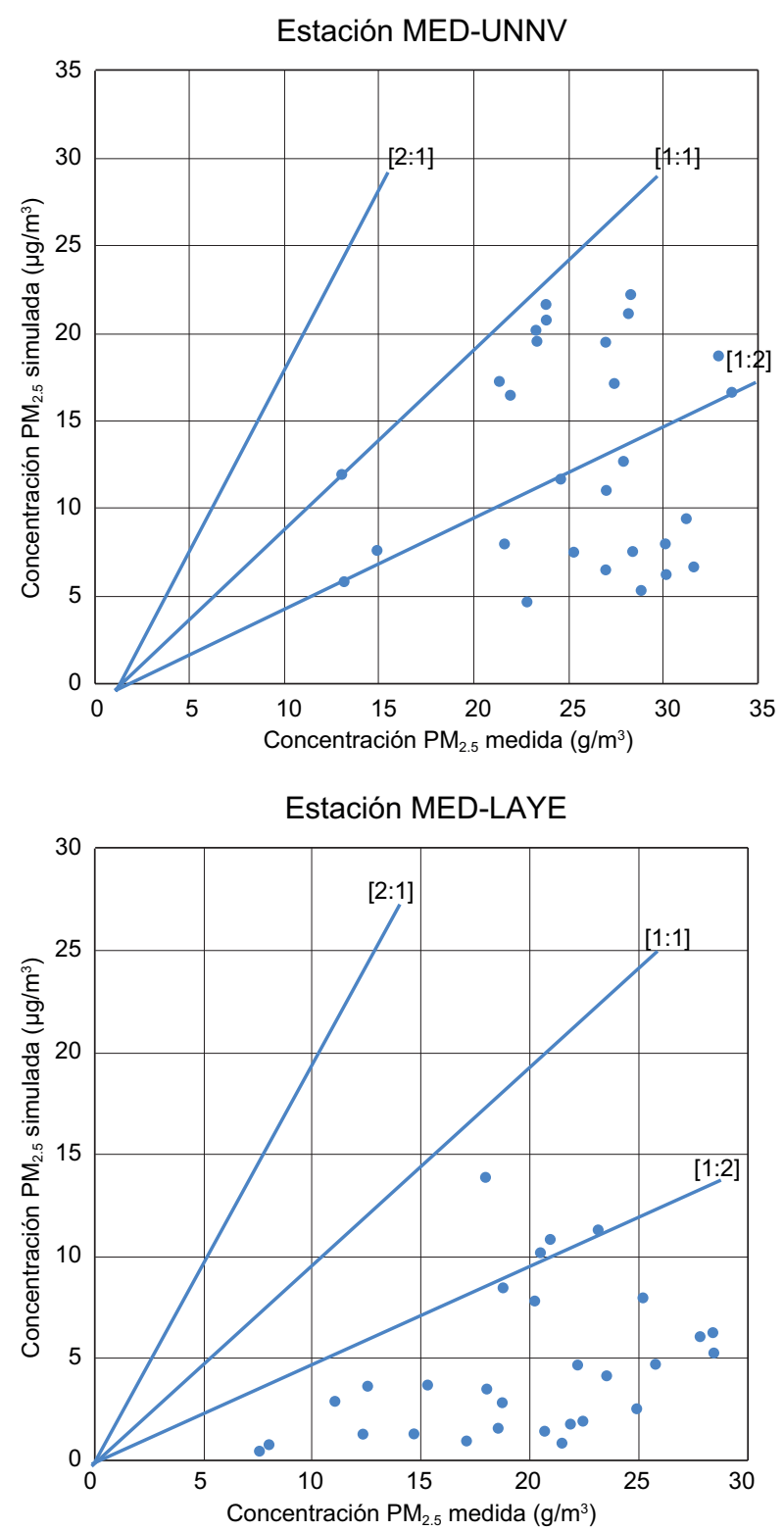

porcentaje de participación en el área de representatividad espacial, el área total y la población de cada comuna. De igual forma, considerando el porcentaje de participación en el área de representatividad espacial y los grupos de edad por género de cada comuna, la tasa de respiración ponderada fue de $16.27 \mathrm{~m}^{3} /$ día.

En el cuadro III se presentan, para cada estación, los datos del área de las comunas que conforman el área de representatividad espacial, su porcentaje de participación, la población total y la densidad poblacional ponderada.

Aunque la densidad poblacional de ambas estaciones fue significativamente diferente, ya que en

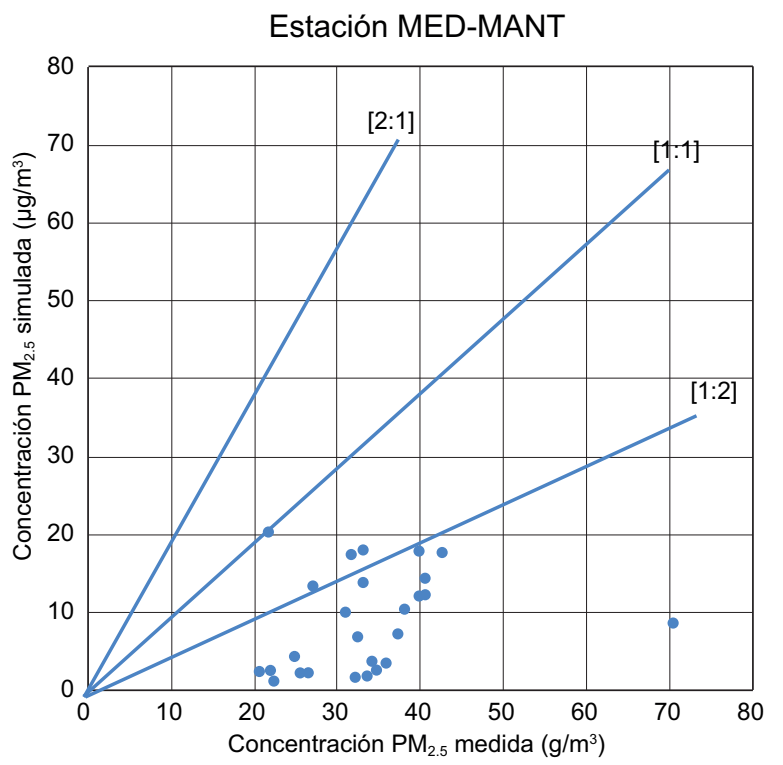

Fig. 5. Comparación de datos observados vs datos simulados con el modelo de calidad del aire comprehensivo con extensiones (CAMx, por sus siglas en inglés) para partículas menores a $2.5 \mu \mathrm{m}\left(\mathrm{PM}_{2.5}\right)$ en las estaciones Universidad Nacional Núcleo el Volador (MED-UNNV), Museo de Antioquia (MED-MANT) y Tanques LAYE (MED-LAYE) la estación MED-UNNV es casi tres veces mayor que en la estación MED-PJIC, la tasa de respiración ponderada de ambas áreas fue similar, por lo que se deduce que la distribución de grupos de edades por género de las comunas que conforman cada área fue porcentualmente similar.

\section{Fracción inhalada}

El valor promedio anual de fracción inhalada (eliminando los datos atípicos) fue de $475 \mathrm{ppm}$ para $\mathrm{PM}_{10}$ y 29 ppm para CO en la estación MED-PJIC, y de 151 ppm para $\mathrm{PM}_{2.5}$ y 64 ppm para NOx en la estación MED-UNNV (Fig. 8). Otra forma de entender 

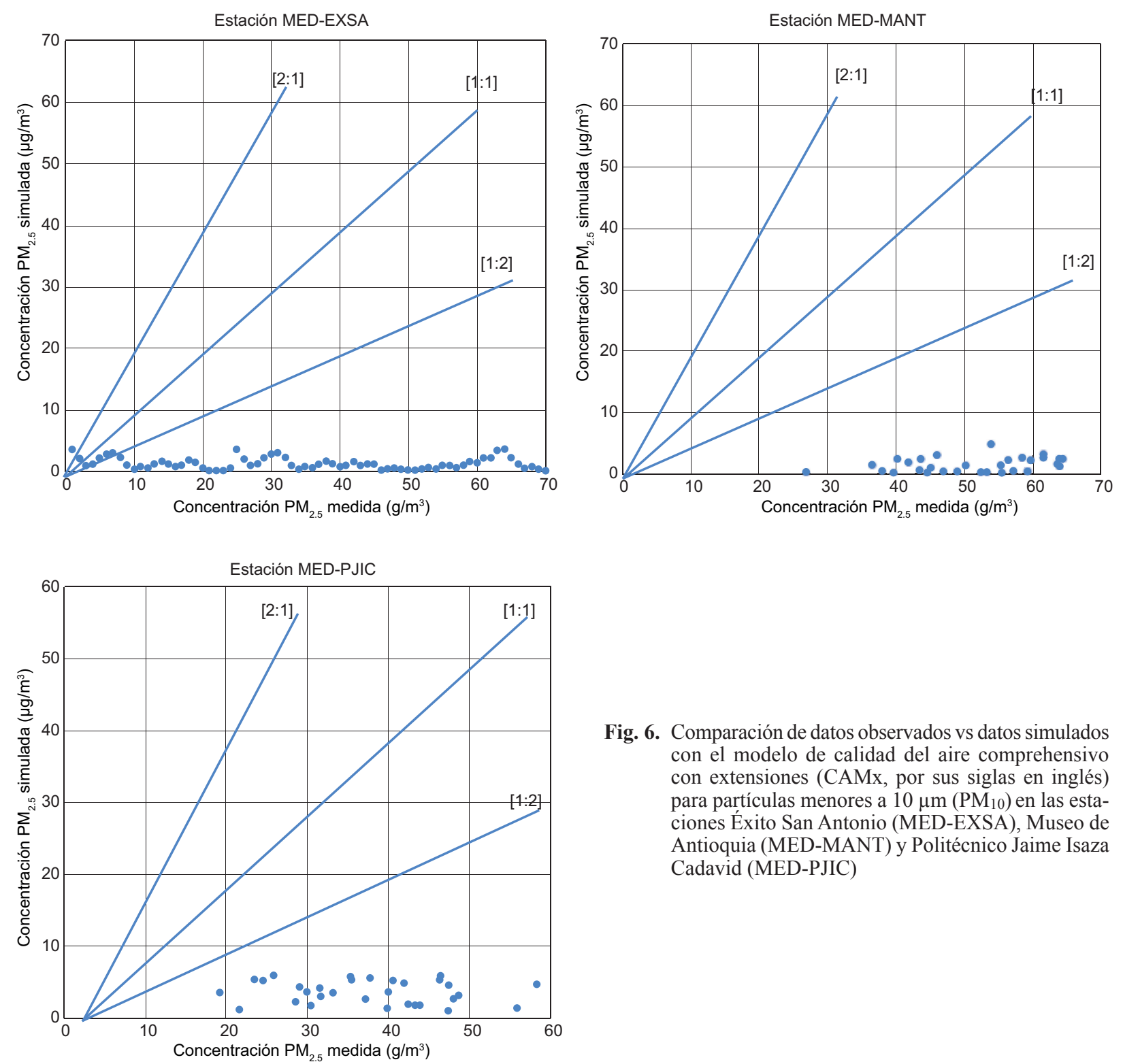

Fig. 6. Comparación de datos observados vs datos simulados con el modelo de calidad del aire comprehensivo con extensiones (CAMx, por sus siglas en inglés) para partículas menores a $10 \mu \mathrm{m}\left(\mathrm{PM}_{10}\right)$ en las estaciones Éxito San Antonio (MED-EXSA), Museo de Antioquia (MED-MANT) y Politécnico Jaime Isaza Cadavid (MED-PJIC)

esta relación es que la población expuesta inhala anualmente, en promedio, 29, 64, 151 y $475 \mathrm{mg}$ por cada kg emitido de $\mathrm{CO}, \mathrm{NO}_{\mathrm{x}}, \mathrm{y}$ partículas $\mathrm{PM}_{2.5} \mathrm{y}$ $\mathrm{PM}_{10}$, respectivamente.

Los datos atípicos (o valores extremos) que correspondieron a los valores que se aislaron y que presentaron inconsistencias con el grupo principal de datos, fueron identificados aplicando la prueba de Tukey a partir de la distribución de los datos en cuartiles y tomando como valores extremos aquellos que se encontraron a 1.5 veces de distancia del valor intercuartil (Amón 2010).

Al comparar estos resultados con los valores de la fracción inhalada para contaminantes de tipo primario de áreas urbanas obtenidos en otros estudios (Cuadro I), se encontró que la fracción inhalada de CO obtenida (29 ppm) es inferior a los valores obtenidos por Marshall et al. (2003) para California (promedio anual de 32-46 ppm) y por Holnicki et al. (2018) para Varsovia (promedio anual de $36 \mathrm{ppm}$ ). Valores más altos de la fracción inhalada para este contaminante fueron reportados por Stevens et al. (2007) para Ciudad de México (promedio mensual de 61-120 ppm) y por Luo et al. (2010) para Hong Kong (promedio anual de 200-300 ppm). 
Estación MED-MANT

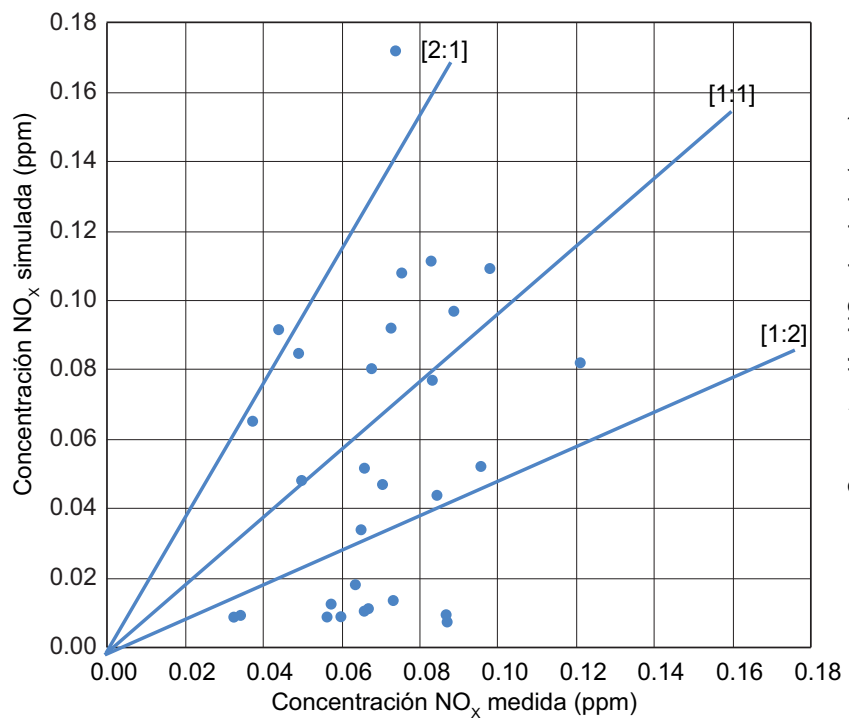

Estación MED-PJIC

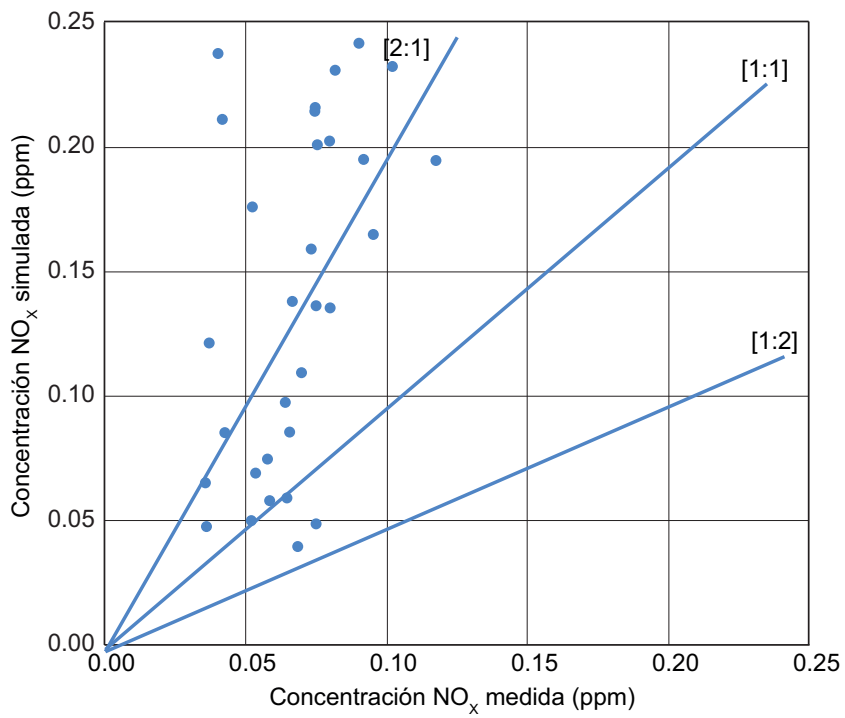

Para el caso del $\mathrm{NO}_{\mathrm{x}}$, el valor de la fracción inhalada obtenido (64 ppm) fue cercano al reportado por Du et al. (2012) para la ciudad de Beijing (70 \pm $32 \mathrm{ppm}$ promedio diario de $24 \mathrm{~h}$ ), pero superior al de Varsovia, donde Tainio et al. (2014) y Holnicki et al. (2018) obtuvieron valores de 13 y 24 ppm como promedio anual, respectivamente.

En cuanto a las partículas $\mathrm{PM}_{2.5}$, hubo similitudes entre el valor de la fracción inhalada obtenido (151 ppm) y el de Beijing (promedio diario de $24 \mathrm{~h}$ de $153 \pm 97 \mathrm{ppm}$ ) reportado por Du et al. (2012). Resultados inferiores fueron reportados para Ciudad de México (promedio anual de 26-120 ppm) según

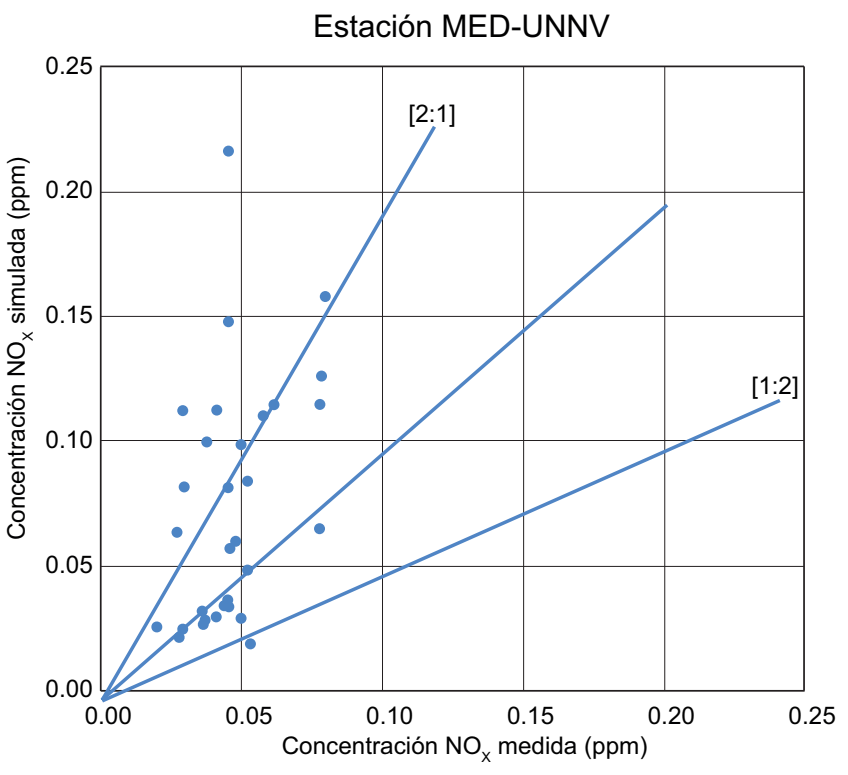

Fig. 7. Comparación de datos observados vs datos simulados con el modelo de calidad del aire comprehensivo con extensiones (CAMx, por sus siglas en inglés) para $\mathrm{NO}_{\mathrm{x}}$ en las estaciones Universidad Nacional Núcleo el Volador (MED-UNNV), Museo de Antioquia (MED-MANT) y Politécnico Jaime Isaza Cadavid (MED-PJIC)
Stevens et al. (2007) y para Varsovia, donde Tainio et al. (2014) y Holnicki et al. (2018) encontraron promedios anuales de 50 y 39 ppm, respectivamente. Estos autores reportaron también para la ciudad de Varsovia valores de la fracción inhalada de partículas $\mathrm{PM}_{10}$ de 51 y 37 ppm (promedio anual), los cuales son inferiores al valor de fracción inhalada obtenido en este estudio (475 ppm).

Al calcular el valor promedio de los cuatro contaminantes $\left(\mathrm{PM}_{10}, \mathrm{PM}_{2.5}, \mathrm{NO}_{\mathrm{x}}\right.$ y $\left.\mathrm{CO}\right)$ combinados, se obtuvo una fracción inhalada de 180 ppm, cifra superior al valor reportado para contaminantes primarios por Stevens et al. (2007) para Ciudad de 
CUADRO III. CÁLCULO DE LA DENSIDAD POBLACIONAL PONDERADA PARA CADA ESTACIÓN DE MEDICIÓN SEGÚN ÁREAS DE REPRESENTATIVIDAD ESPACIAL

\begin{tabular}{|c|c|c|}
\hline Estación MED-PJIC & $\begin{array}{l}\text { Comuna } \\
\text { Área total de la comuna }\left(\mathbf{k m}^{2}\right) \\
\text { Participación en área de representatividad espacial (\%) } \\
\text { Población total (personas) } \\
\left.\text { Densidad poblacional ponderada (personas } / \mathrm{km}^{2}\right)\end{array}$ & $\begin{array}{l}\text { Poblado } \\
14.74 \\
100 \\
128839 \\
8742\end{array}$ \\
\hline Estación MED-UNNV & $\begin{array}{l}\text { Comuna } \\
\text { Área total de la comuna }\left(\mathbf{k m}^{2}\right) \\
\text { Participación en área de representatividad espacial (\%) } \\
\text { Población total (personas) } \\
\text { Comuna } \\
\text { Área total de la comuna }\left(\mathbf{k m}^{2}\right) \\
\text { Participación en área de representatividad espacial (\%) } \\
\text { Población total (personas) } \\
\text { Comuna } \\
\text { Área total de la comuna }\left(\mathbf{k m}^{2}\right) \\
\text { Participación en área de representatividad espacial (\%) } \\
\text { Población total (personas) } \\
\text { Comuna } \\
\text { Área total de la comuna }\left(\mathbf{k m}^{2}\right) \\
\text { Participación en área de representatividad espacial (\%) } \\
\text { Población total (personas) } \\
\text { Comuna } \\
\text { Área total de la comuna (km²) } \\
\text { Participación en área de representatividad espacial (\%) } \\
\text { Población total (personas) } \\
\left.\text { Densidad poblacional ponderada (personas } / \mathrm{km}^{2}\right)\end{array}$ & $\begin{array}{l}\text { Castilla } \\
6.18 \\
74.5 \\
149751 \\
\text { Aranjuez } \\
4.97 \\
12.1 \\
162252 \\
\text { Robledo } \\
9.46 \\
10.2 \\
171660 \\
\text { Doce Octubre } \\
3.99 \\
2.5 \\
193657 \\
\text { La Candelaria } \\
7.49 \\
0.8 \\
85505 \\
25158\end{array}$ \\
\hline
\end{tabular}

MED-PJIC: Politécnico Jaime Isaza Cadavid, MED-UNNV: Universidad Nacional Núcleo el Volador

México (promedio anual de 40-120 ppm), pero inferior a la fracción inhalada de $220 \mathrm{ppm}$ (promedio anual) reportada por Apte et al. (2012) para la ciudad de Medellín.

Acorde con el inventario de emisiones, la contribución por tipo de fuentes correspondió en su mayoría a fuentes de tipo móviles. Los valores de fracción inhalada para los contaminantes $\mathrm{PM}_{10}, \mathrm{PM}_{2.5}$ y CO no tuvieron aporte de fuentes fijas, mientras que en los $\mathrm{NO}_{\mathrm{x}}$, el $7 \%$ correspondió a este tipo de fuente (Fig. 9). Esta distribución está en concordancia con el último inventario de emisiones atmosféricas del Valle de Aburrá en el que se reporta que la mayor parte de las emisiones de todos los contaminantes $\mathrm{CO}, \mathrm{NO}_{\mathrm{x}}$, VOC y $\mathrm{PM}_{2.5}$ son generadas por fuentes móviles.

La problemática de emisión de este tipo de contaminantes por fuentes móviles en la región se asocia principalmente con la calidad de los combustibles diésel y gasolina disponibles (debido a su contenido de azufre), la tecnología del parque automotor que determina la posibilidad de cumplimiento de estándares de emisión menos contaminantes como
Euro 4 y 5, el crecimiento del parque automotor (principalmente de motocicletas y automóviles), así como la antigüedad de los vehículos tipo camiones y volquetas, que a pesar de ser un número reducido en comparación con las otras categorías, son responsables de la mayoría de las emisiones de $\mathrm{CO}, \mathrm{NO}_{\mathrm{x}} \mathrm{y}$ $\mathrm{PM}_{2.5}$ (AMVA-UPB 2017a).

Al realizar los cálculos de los valores de la fracción inhalada utilizando las tasas de respiración ponderadas por grupos de edad (entre los 0 y 4,5 a 14, 15 a 59 y 60 o más años), se identificó que para aquellos grupos en los cuales la tasa de respiración fue mayor, la fracción inhalada también lo fue (Cuadro IV).

Se utilizaron los valores promedio horarios de manera diaria, según día de la semana y por mes, a partir de las cifras de concentración y emisión de contaminantes, con el fin de evaluar el comportamiento de los valores de la fracción inhalada. En este caso no se eliminaron los datos atípicos que se encuentran de color rojo en las gráficas. Además, se presenta el valor promedio calculado sin atípicos en forma de línea punteada de color azul. 

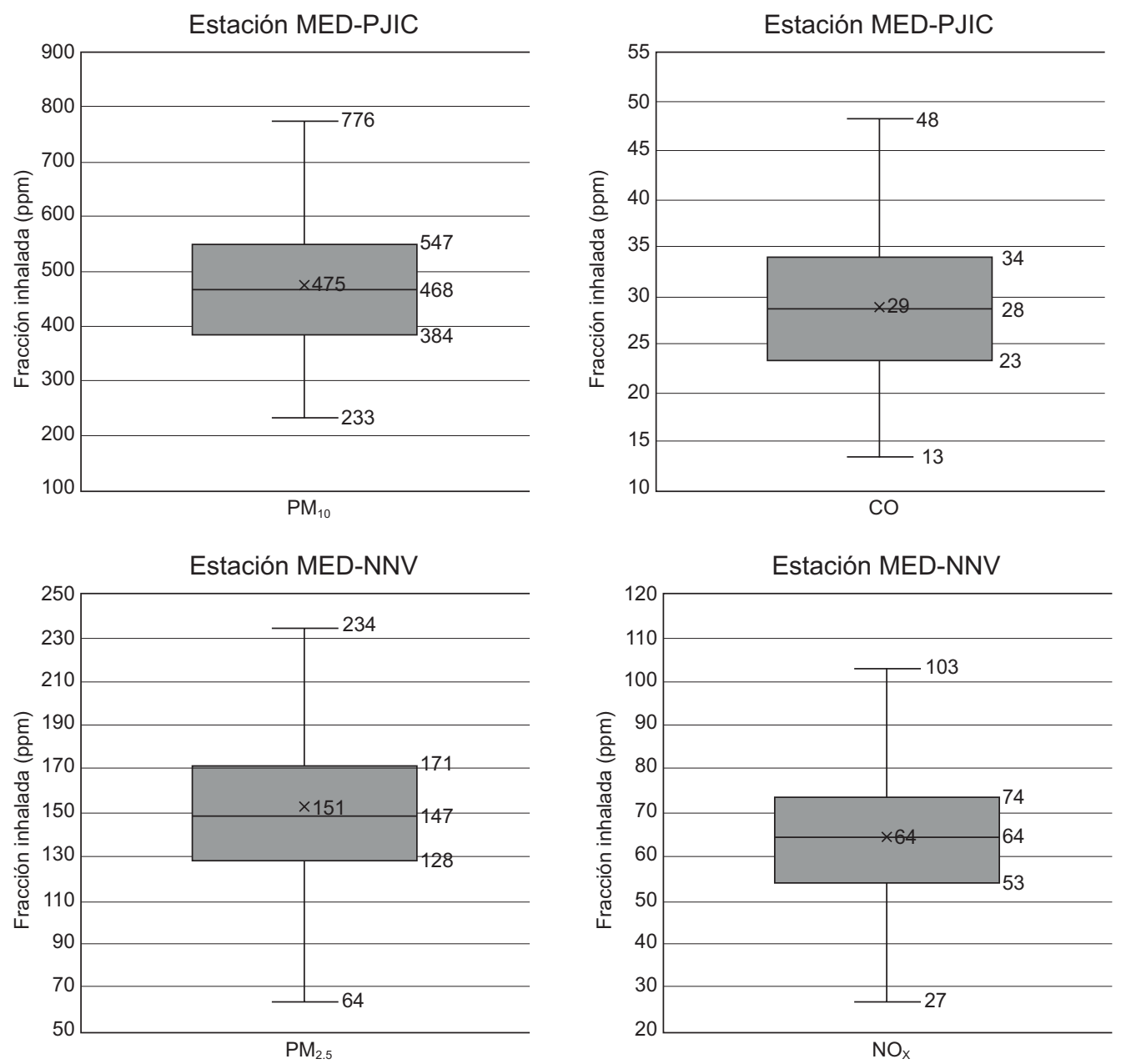

Fig. 8. Valor promedio anual de la fracción inhalada para partículas menores a $10 \mu \mathrm{m}\left(\mathrm{PM}_{10}\right)$ y $\mathrm{CO}$ en la estación Politécnico Jaime Isaza Cadavid (MED-PJIC), y para partículas menores a $2.5 \mu \mathrm{m}\left(\mathrm{PM}_{2.5}\right)$ y $\mathrm{NO}_{\mathrm{x}}$ en la estación Universidad Nacional Núcleo el Volador (MED-UNNV).

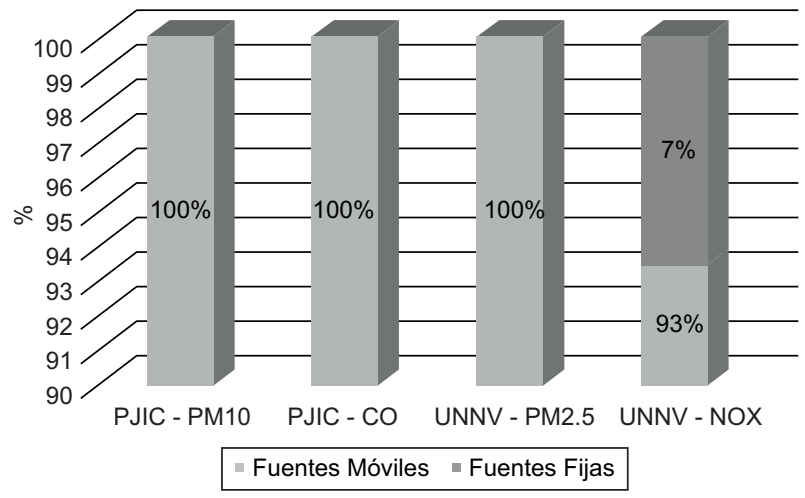

Fig. 9. Contribución por tipo de fuentes (fijas y móviles) a los valores de la fracción inhalada para partículas menores a $10 \mu \mathrm{m}\left(\mathrm{PM}_{10}\right)$ y CO en la estación Politécnico Jaime Isaza Cadavid (MED-PJIC), y para partículas menores a 2.5 $\mu \mathrm{m}\left(\mathrm{PM}_{2.5}\right)$ y $\mathrm{NO}_{\mathrm{x}}$ en la estación Universidad Nacional Núcleo el Volador (MED-UNNV)
En el análisis de los valores horarios de la fracción inhalada (Fig. 10) se presentan paralelamente los valores de emisión y concentración (también horarios) para entender mejor la relación entre estas dos variables. Los máximos valores de la fracción inhalada ocurren cuando la emisión de contaminantes es significativamente inferior a la concentración. Esto ocurre en las horas del día donde hay menos emisiones (de 01:00 a 06:00 y de 21:00 a 24:00 horas), las cuales son liberadas mayoritariamente por las fuentes móviles, de acuerdo con los volúmenes vehiculares típicos de la ciudad (Posada et al. 2011). Por otro lado, la ausencia de radiación solar dificultó la dispersión de contaminantes con un consecuente aumento en los valores de concentración.

Las variaciones en los estimados diarios de la fracción inhalada (Fig. 11) se relacionan de forma 
CUADRO IV. VALORES PROMEDIO DE LA FRACCIÓN INHALADA POR GRUPOS DE EDADES

\begin{tabular}{|c|c|c|c|c|c|c|}
\hline \multirow{3}{*}{$\begin{array}{l}\text { Grupo de edad } \\
\text { (años) }\end{array}$} & \multicolumn{3}{|c|}{ Estación MED-PJIC } & \multicolumn{3}{|c|}{ Estación MED-UNNV } \\
\hline & \multirow{2}{*}{$\begin{array}{c}\text { Tasa de respiración } \\
\left(\mathrm{m}^{3} / \text { día }\right)\end{array}$} & \multicolumn{2}{|c|}{$\begin{array}{c}\text { Fracción inhalada (promedio } \\
\text { en ppm) }\end{array}$} & \multirow{2}{*}{$\begin{array}{l}\text { Tasa de respiración } \\
\qquad\left(\mathrm{m}^{3} / \text { día }\right)\end{array}$} & \multicolumn{2}{|c|}{$\begin{array}{c}\text { Fracción inhalada (promedio } \\
\text { en ppm) }\end{array}$} \\
\hline & & $\mathrm{PM}_{10}$ & $\mathrm{CO}$ & & $\mathrm{PM}_{2.5}$ & $\mathrm{NO}_{\mathrm{x}}$ \\
\hline $0-4$ & 11.68 & 337.99 & 20.45 & 11.68 & 108.28 & 46.02 \\
\hline $5-14$ & 13.57 & 392.67 & 23.76 & 13.54 & 125.58 & 53.37 \\
\hline $15-59$ & 17.60 & 509.26 & 30.81 & 17.51 & 162.32 & 68.98 \\
\hline 60 y más & 14.46 & 418.64 & 25.33 & 14.50 & 134.44 & 57.13 \\
\hline
\end{tabular}

MED-PJIC: Politécnico Jaime Isaza Cadavid, MED-UNNV: Universidad Nacional Núcleo el Volador

directa con los promedios diarios de concentración, dado que el valor de emisión diario es constante. Los valores pico (identificados como datos atípicos) de $\mathrm{PM}_{2.5}$ y $\mathrm{PM}_{10}$ se presentan finalizando febrero y durante marzo, lo cual corresponde a una época de transición entre la temporada seca y la húmeda. Ésta genera condiciones meteorológicas poco favorables para la dispersión de los contaminantes con el respectivo aumento de los valores de concentración, ocasionando los episodios de contaminación ambiental atmosférica declarados en la región (AMVA 2016, IDEAM 2017b, AMVA 2018).

Para el caso del $\mathrm{NO}_{\mathrm{x}}$ y el $\mathrm{CO}$, los valores pico se presentaron mayoritariamente entre los meses de septiembre y noviembre. El aumento de los valores de concentración en este período se asocia con una condición de lluvia escasa que ocurrió durante el segundo semestre del año, presentándose reducciones de hasta el $50 \%$ de la lluvia esperada en los meses que son típicamente lluviosos (IDEAM 2015).

Los valores de la fracción inhalada por mes (Fig. 12) concuerdan con el comportamiento de los valores diarios de la fracción inhalada. Las mayores cifras se presentaron en los meses en que ocurren valores pico de concentración, siendo éstos marzo para las partículas $\mathrm{PM}_{10}$ y $\mathrm{PM}_{2.5}$, el periodo octubre-diciembre para el $\mathrm{NO}_{\mathrm{x}} \mathrm{y}$ octubre para el $\mathrm{CO}$.

En la distribución de los valores de fracción inhalada por día de la semana (Fig. 13), el domingo reporta un menor valor para todos los contaminantes asociado con una menor cantidad de emisiones, siguiendo el mismo patrón descrito en la variación por horas del día.

\section{CONCLUSIONES}

El método de CSF utilizado para evaluar la representatividad espacial de las estaciones de medición de calidad del aire permitió obtener las áreas para los contaminantes $\mathrm{PM}_{10}$ y $\mathrm{CO}$ en la estación MEDPJIC, y $\mathrm{PM}_{2.5}$ y $\mathrm{NO}_{\mathrm{x}}$ en la estación MED-UNNV. Este método tiene alta dependencia de la resolución espacial de las celdas que se utilice, ya que las áreas de representatividad de algunas estaciones de medición y contaminantes pueden ser menores a $1 \mathrm{~km}^{2}$ y/o no coincidir con la distribución espacial de las celdas del modelo de emisiones atmosféricas del área metropolitana del Valle de Aburrá que fue utilizado.

La evaluación del desempeño (incertidumbre) de los datos de concentración empleados para la determinación de áreas de representatividad espacial de las estaciones de medición, obtenida de simulaciones del modelo CAMx, indica que los mejores resultados se obtuvieron con el CO. Para el contaminante $\mathrm{PM}_{2.5}$, el modelo tendió a subestimar los valores de concentración. En el caso del $\mathrm{NO}_{\mathrm{x}}$, contaminante altamente reactivo compuesto por las especies primarias óxido nítrico (NO) y dióxido de nitrógeno $\left(\mathrm{NO}_{2}\right)$, el modelo tuvo en general un buen desempeño con tendencia a subestimar algunos valores. Con el contaminante $\mathrm{PM}_{10}$ el rendimiento fue bastante bajo, ya que el proceso químico de aerosoles estuvo enfocado a partículas finas y este contaminante pertenece al tipo de partículas gruesas.

La densidad poblacional estimada en personas/ $\mathrm{km}^{2}$ varió significativamente (en una proporción de 1:3) entre áreas de representatividad espacial, siendo mayor en la estación MED-UNNV que en la estación MED-PJIC. Las tasas de respiración ponderadas de ambas áreas fueron similares, lo cual indica que la distribución de grupos de edad por género de las comunas también es porcentualmente similar.

Los resultados de fracción inhalada de $\mathrm{NO}_{\mathrm{x}} \mathrm{y}$ $\mathrm{PM}_{2.5}$ ubican las áreas de representatividad espacial evaluadas en la ciudad de Medellín en niveles comparables para estos contaminantes a los reportados para la ciudad de Beijing. En el caso del contaminante 

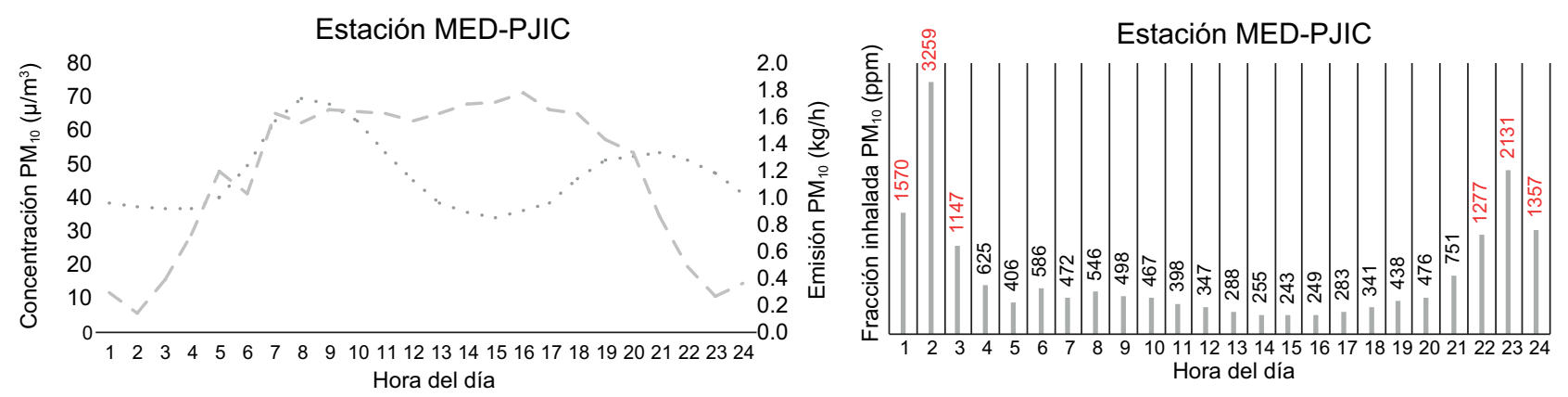

Estación MED-PJIC

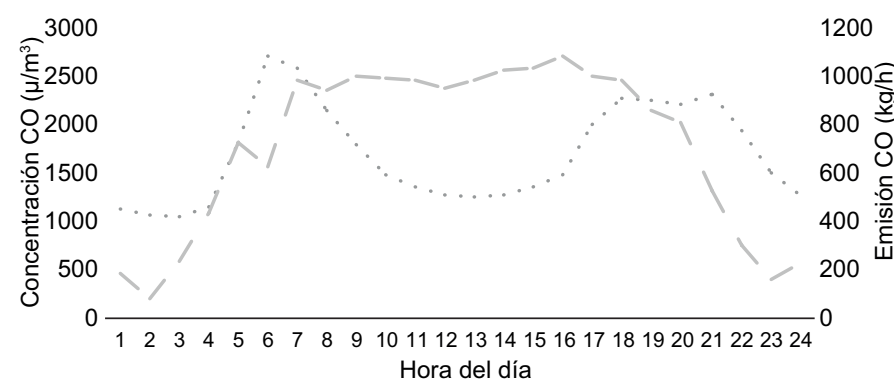

Estación MED-PJIC
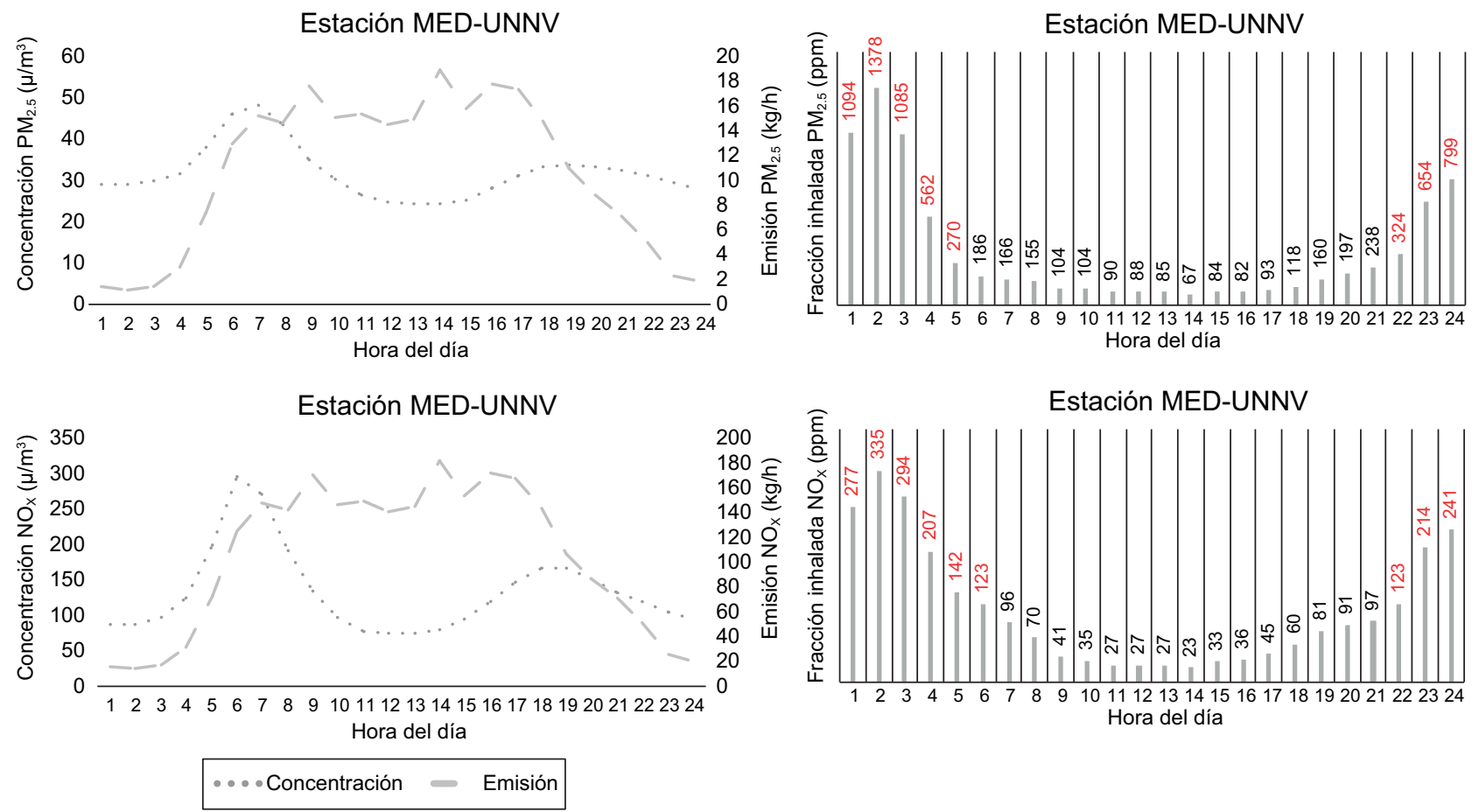

Fig. 10. Valores horarios de concentración, emisión y fracción inhalada para partículas menores a $10 \mu \mathrm{m}\left(\mathrm{PM}_{10}\right)$ y $\mathrm{CO}$ en la estación Politécnico Jaime Isaza Cadavid (MED-PJIC), y para partículas menores a $2.5 \mu \mathrm{m}\left(\mathrm{PM}_{2.5}\right)$ y $\mathrm{NO}_{\mathrm{x}}$ en la estación Universidad Nacional Núcleo el Volador (MED-UNNV)

$\mathrm{CO}$, los valores de fracción inhalada fueron inferiores a los reportados para ciudades como California, Varsovia, Ciudad de México y Hong Kong, mientras que para el contaminante $\mathrm{PM}_{10}$ los valores fueron muy superiores a los tomados como referencia. $\mathrm{Si}$ se agrupan los cuatro contaminantes $\left(\mathrm{PM}_{10}, \mathrm{PM}_{2.5}\right.$, $\mathrm{NO}_{\mathrm{x}}$ y $\mathrm{CO}$ ) en la denominación de contaminantes primarios, el valor promedio de fracción inhalada es superior al de California, pero inferior al calculado por otros autores para Medellín. 
Estación MED-PJIC

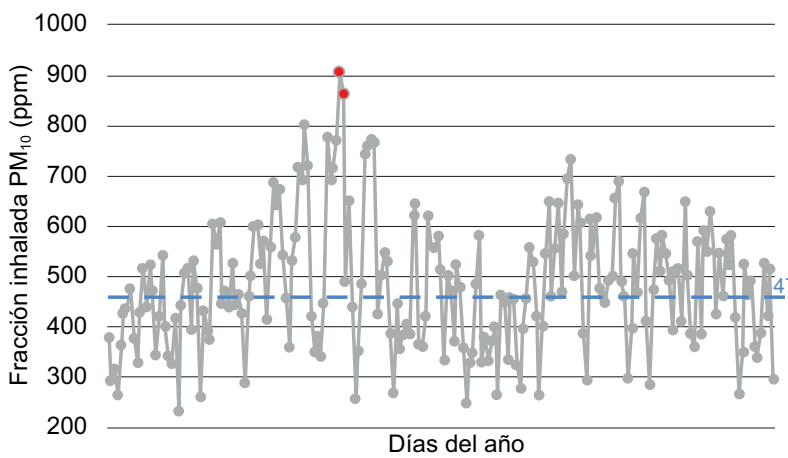

Estación MED-UNNV

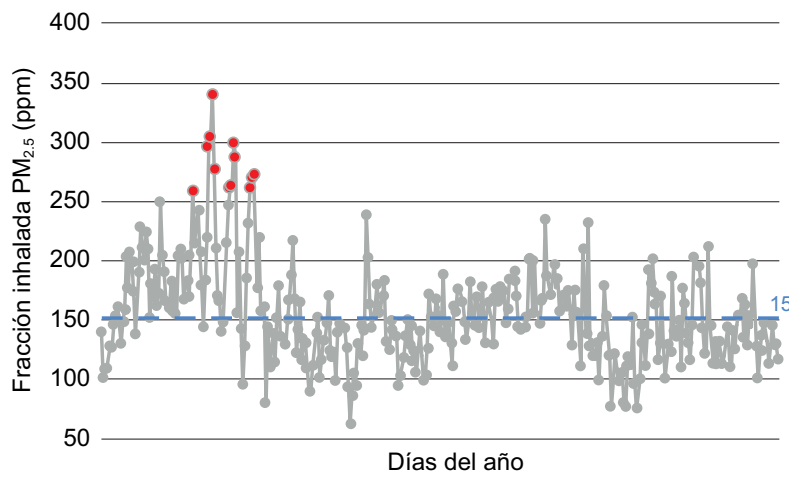

Estación MED-PJIC

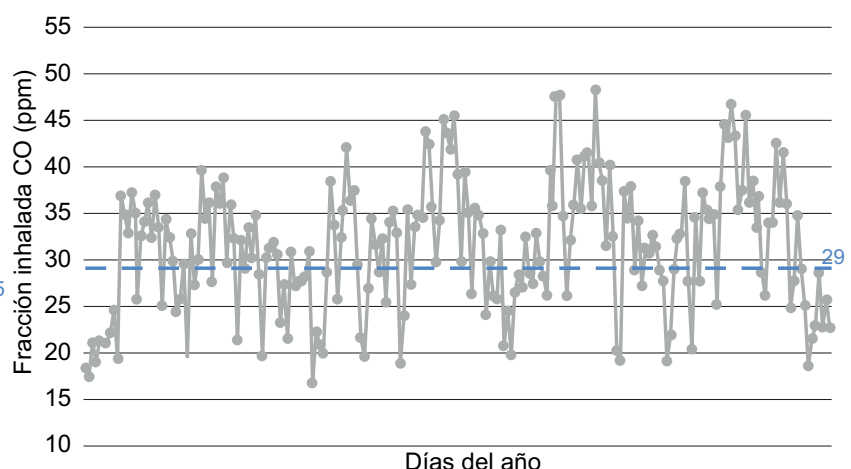

Estación MED-UNNV

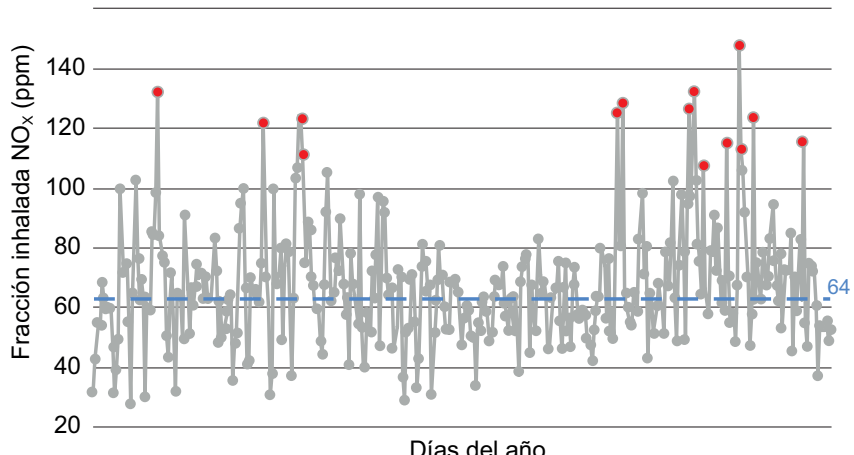

Fig. 11.Valores diarios de la fracción inhalada para partículas menores a $10 \mu \mathrm{m}\left(\mathrm{PM}_{10}\right)$ y $\mathrm{CO}$ en la estación Politécnico Jaime Isaza Cadavid (MED-PJIC), y para partículas menores a $2.5 \mu \mathrm{m}\left(\mathrm{PM}_{2.5}\right)$ y $\mathrm{NO}_{\mathrm{x}}$ en la estación Universidad Nacional Núcleo el Volador (MED-UNNV)

Estación MED-PJIC

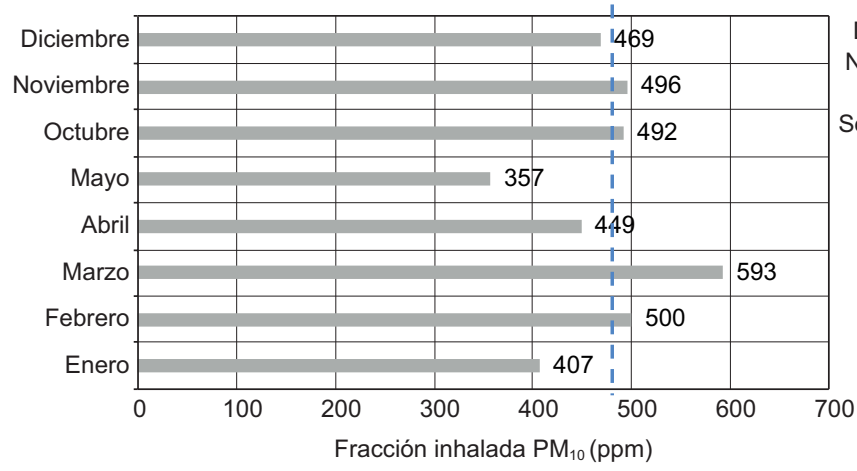

Estación MED-UNNV

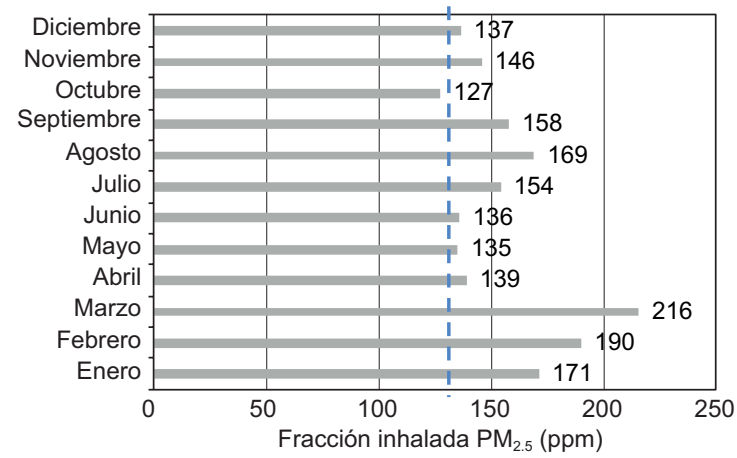

Estación MED-PJIC

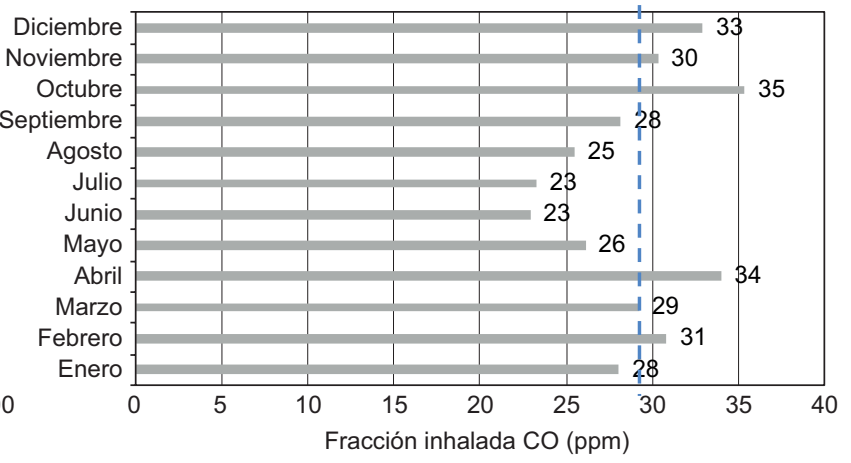

Estación MED-UNNV

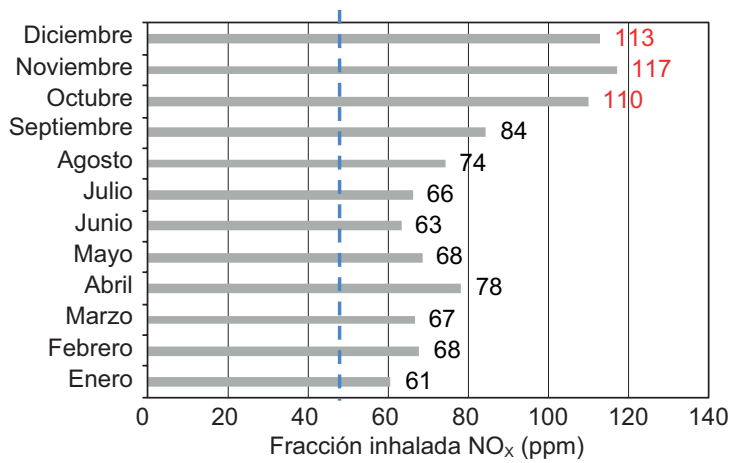

Fig. 12.Valores mensuales de la fracción inhalada para partículas menores a $10 \mu \mathrm{m}\left(\mathrm{PM}_{10}\right)$ y $\mathrm{CO}$ en la estación Politécnico Jaime Isaza Cadavid (MED-PJIC), y para partículas menores a $2.5 \mu \mathrm{m}\left(\mathrm{PM}_{2.5}\right)$ y $\mathrm{NO}_{\mathrm{x}}$ en la estación Universidad Nacional Núcleo el Volador (MED-UNNV) 
Estación MED-PJIC

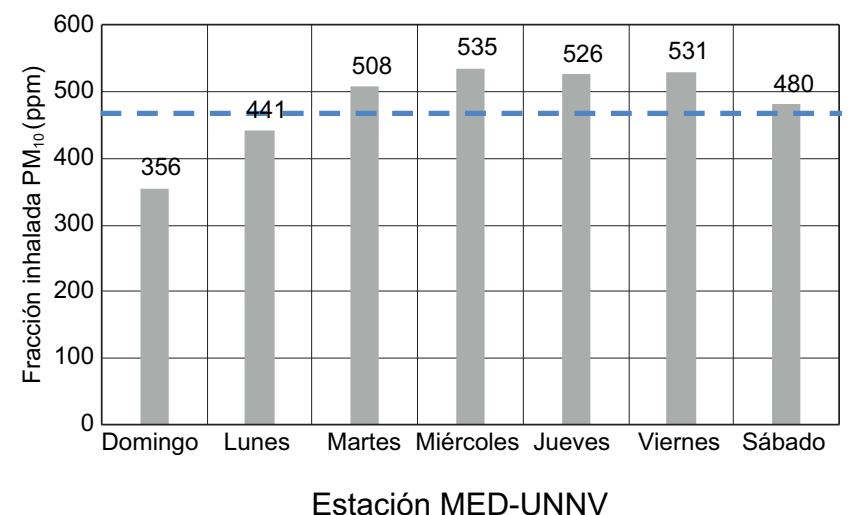

Estación MED-UNNV

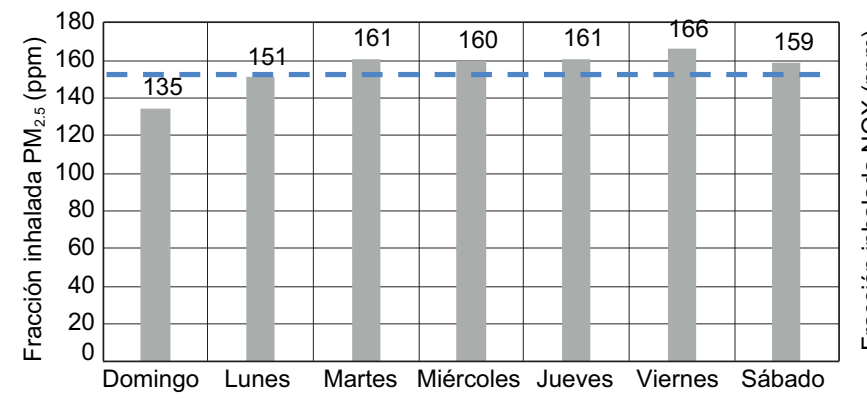

Estación MED-PJIC

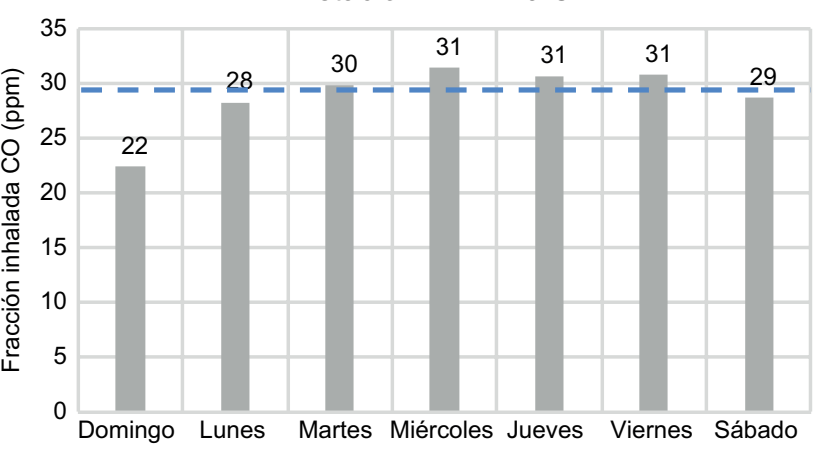

Estación MED-UNNV

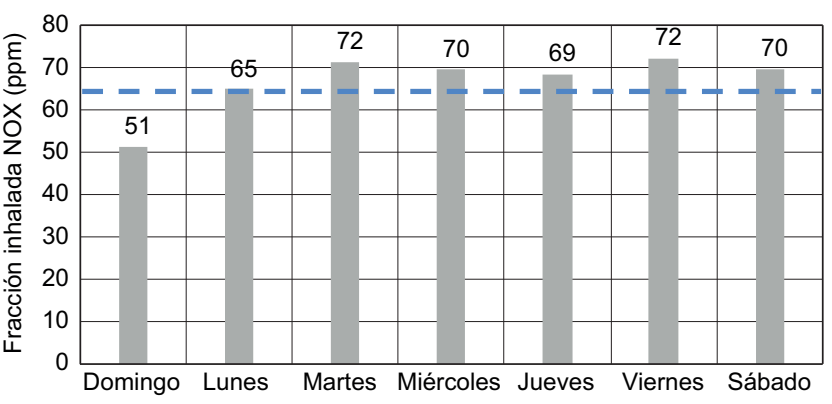

Fig. 13.Valores por día de la semana de la fracción inhalada para partículas menores a $10 \mu \mathrm{m}\left(\mathrm{PM}_{10}\right)$ y CO en la estación Politécnico Jaime Isaza Cadavid (MED-PJIC), y para partículas menores a $2.5 \mu \mathrm{m}\left(\mathrm{PM}_{2.5}\right)$ y $\mathrm{NO}_{\mathrm{x}}$ en la estación Universidad Nacional Núcleo el Volador (MED-UNNV)

De acuerdo con el inventario de emisiones, los valores de fracción inhalada obtenidos corresponden en su totalidad a fuentes de tipo móviles, a excepción de los $\mathrm{NO}_{\mathrm{x}}$ en los cuales hay una pequeña contribución de fuentes fijas.

Existe una alta variabilidad en los resultados obtenidos dependiendo de la forma en que se empleen los valores de tasa de respiración, concentración y emisión. Para los grupos de edad en los cuales la tasa de respiración fue mayor, la fracción inhalada también lo fue. Los máximos valores horarios de fracción inhalada se presentaron cuando la emisión de contaminantes fue significativamente inferior a la concentración. La variabilidad de los valores diarios de fracción inhalada se relacionó de forma directa con los promedios diarios de concentración.

\section{AGRADECIMIENTOS}

Al equipo de trabajo del Grupo de Investigaciones Ambientales-Línea de Estudios Atmosféricos de la Universidad Pontificia Bolivariana, Sede Medellín, por su acompañamiento y soporte apoyo en la obtención de los resultados de esta investigación.

\section{REFERENCIAS}

Amón I. (2010). Guía metodológica para la selección de técnicas de depuración de datos. Tesis de Maestría. Facultad de Minas, Universidad Nacional de Colombia. Medellín, Colombia, 120 pp.

AMVA (2007). Plan Integral de Desarrollo Metropolitano METRÓPOLI 2008-2020. Área Metropolitana del Valle de Aburrá. Informe. Medellín, Colombia. 280 pp.

AMVA (2016). Resolución metropolitana $\mathrm{N}^{\circ} 000379$ de 2016. Por la cual se declara el nivel de contingencia atmosférica en la jurisdicción del Área Metropolitana del Valle de Aburrá y se toman otras determinaciones. Área Metropolitana del Valle de Aburrá. Medellín, Colombia, 18 de marzo.

AMVA-EAFIT (2017). Informe anual 2017. Calidad del aire. Área Metropolitana del Valle de Aburrá y Universidad Escuela de Administración y FinanzasInstituto Tecnológico. Informe. Medellín, Colombia, $79 \mathrm{pp}$.

AMVA-UPB (2017a). Actualización inventario de emisiones atmosféricas del Valle de Aburrá, año 2015. Área Metropolitana del Valle de Aburrá y Universidad Pontificia Bolivariana. Informe. Medellín, Colombia, 90 pp. 
AMVA-UPB (2017b). Sistema de modelización atmosférica: modelo de calidad del aire. Área Metropolitana del Valle de Aburrá y Universidad Pontificia Bolivariana. Informe. Medellín, Colombia, $82 \mathrm{pp}$.

AMVA (2018). Resolución metropolitana $\mathrm{N}^{\circ} 00-000475$ de 2018. Por la cual se declara el nivel de alerta para el control de la contaminación atmosférica en la jurisdicción del Área Metropolitana del Valle de Aburrá. Área Metropolitana del Valle de Aburrá. Medellín, Colombia, 6 de marzo.

AMVA-UPB (2018a). Sistema de Información Metropolitano de la Calidad del Aire (SIMECA). Área Metropolitana del Valle de Aburrá-Universidad Pontificia Bolivariana [en línea]. http://sigaire.upb.edu.co/ simeca/index.php 10/03/2018

AMVA-UPB (2018b). Modelo de Emisiones Atmosféricas del Valle de Aburrá (MODEAM). Área Metropolitana del Valle de Aburrá-Universidad Pontificia Bolivariana [en línea]. http://modemed.upb.edu.co/zigma2/index. php?option $=$ com_content\&view $=$ category\&layout $=\mathrm{b}$ $\log \&$ id=90\&Itemid= 499 10/03/2018

AMVA-UPB (2018c). Sistema Inteligente de Información para el Manejo de la Calidad del Aire en el Valle de Aburrá. Área Metropolitana del Valle de AburráUniversidad Pontificia Bolivariana [en línea]. http:// modemed.upb.edu.co/zigma2/index.php?option $=$ com content $\&$ view $=$ category\&layout $=$ blog $\&$ id $=93 \&$ Item $\mathrm{id}=50010 / 03 / 2018$

Apte J.S., Bombrun E., Marshall J.D. y Nazaroff W.W. (2012). Global intraurban intake fractions for primary air pollutants from vehicles and other distributed sources. Environ. Sci. Technol. 46 (6), 3415-3423. http://doi.org/10.1021/es204021h

Bennett D.H., McKone T.E., Evans J.S., Nazaroff W.W., Margni M.D., Jolliet O. y Smith K.R. (2002). Defining intake fraction. Environ. Sci. Technol. 36 (9), 206A211A. http://doi.org/10.1021/es0222770

CE (2008). Directiva 2008/50/CE de 21 de mayo de 2008 relativa a la calidad del aire ambiente y a una atmósfera más limpia en Europa. Parlamento Europeo y Consejo de la Unión Europea. Estrasburgo, Francia, 21 de mayo.

Du X., Wu Y., Fu L., Wang S., Zhang S. y Hao J. (2012). Intake fraction of $\mathrm{PM}_{2.5}$ and $\mathrm{NO}_{\mathrm{x}}$ from vehicle emissions in Beijing based on personal exposure data. Atmos. Environ. 57, 233-243. http://doi.org/10.1016/j. atmosenv.2012.04.046

Foy B., Lei W., Zavala M., Volkamer R., Samuelsson J., Mellqvist J., Galle B., Martínez A.-P., Grutter M., Retama A. y Molina L.T. (2007). Modelling constraints on the emission inventory and on vertical dispersion for $\mathrm{CO}$ and $\mathrm{SO}_{2}$ in the Mexico City Metropolitan Area using Solar FTIR and zenith sky UV spectroscopy. At- mos. Chem. Phys. 7, 781-801. http://doi.org/10.5194/ acpd-6-6125-2006

Humbert S., Marshall J.D., Shaked S., Spadaro J.V., Nishioka Y., Preiss P., McKone T.E., Horvath A. y Jolliet O. (2011). Intake fraction for particulate matter: recommendations for life cycle impact assessment. Environ. Sci. Technol. 45, 4808-4816. http://doi.org/10.1021/ es $103563 z$

Holnicki P., Nahorski Z. y Kałuszko A. (2018). Intake Fraction (iF) assessment in an urban area. IFAC Papersonline 51, 79-84. http://doi.org/10.1016/j.ifacol.2018.06.214

$\operatorname{IDEAM}(2002)$. Auditoría a la red de monitoreo de calidad del aire de Bogotá-Anexo 9: Guía para la utilización de modelos de la calidad del aire. Instituto de Hidrología, Meteorología y Estudios Ambientales. Informe. Bogotá D.C., Colombia, 36 pp.

IDEAM (2015). Anuario climatológico 2015. Instituto de Hidrología, Meteorología y Estudios Ambientales. Informe. Bogotá D.C., Colombia, 365 pp.

IDEAM (2017a). Informe del estado de la calidad del aire en Colombia 2016. Instituto de Hidrología, Meteorología y Estudios Ambientales. Informe. Bogotá D.C., Colombia, 106 pp.

IDEAM (2017b). Tercer boletín sobre contaminación atmosférica. Instituto de Hidrología, Meteorología y Estudios Ambientales. Boletín. Bogotá D.C., Colombia, $11 \mathrm{pp}$.

Jeričević A. y Večenaj Ž. (2009). Improvement of vertical diffusion analytic schemes under stable atmospheric conditions. Bound.-Lay. Meteorol. 131, 293-307. http://doi.org/10.1007/s10546-009-9367-5

Lai A.C.K, Thatcher T.L. y Nazaroff W.W. (2000). Inhalation transfer factors for air pollution health risk assessment. JAPCA J. Air Waste. Ma. 50, 1688-1699. http:// doi.org/10.1080/10473289.2000.10464196

Luo Z., Li Y. y Nazaroff W.W. (2010). Intake fraction of nonreactive motor vehicle exhaust in Hong Kong. Atmos. Environ. 44, 1913-1918. http://doi.org/10.1016/j. atmosenv.2010.02.016

MADS (2012). Diagnóstico Nacional de Salud Ambiental. Ministerio de Ambiente y Desarrollo Sostenible. Informe. Bogotá D.C., Colombia, 368 pp.

MADS (2017). Resolución 2254 de 2017. Por la cual se adopta la norma de calidad del aire ambiente y se dictan otras disposiciones. Ministerio de Ambiente y Desarrollo Sostenible. Bogotá D.C., Colombia, 1 de noviembre.

Marshall J.D. y Nazaroff W.W. (2002). Risk assessment of diesel-fired backup electric generators operating in California [en línea]. https://depts.washington. edu/airqual/reports/MARSHALL\%20and\%20NAZAROFF\%20-\%20Diesel\%20BUGs\%20Health\%20 Risk\%20Assessment.pdf 12/07/2018 
Marshall J.D., Riley W.J., McKone T.E. y Nazaroff W.W. (2003). Intake fraction of primary pollutants: Motor vehicle emissions in the South Coast Air Basin. Atmos. Environ. 37, 3455-3468. http://doi.org/10.1016/ S1352-2310(03)00269-3

Marshall J.D. y Nazaroff W.W. (2006). Intake fraction. En: Exposure analysis (Ott W.R., Steinemann A.C. y Wallace L.A., Eds.). Taylor and Francis, Boca Raton, Florida, EUA, pp. 237-252.

MAVDT (2008). Manual de operación de sistemas de vigilancia de la calidad del aire. Ministerio de Ambiente, Vivienda y Desarrollo Territorial. Protocolo. Bogotá D.C., Colombia, 287 pp.

MAVDT (2010). Manual de diseño de sistemas de vigilancia de la calidad del aire. Ministerio de Ambiente, Vivienda y Desarrollo Territorial. Protocolo. Bogotá D.C., Colombia, 135 pp.

Municipio de Medellín-DANE (2011). Proyecciones de Población 2006-2015. Municipio de Medellín-Departamento Nacional de Estadísticas. Informe. Medellín, Colombia, 199 pp.

Nappo C., Caneill J., Furman R., Gifford F., Kaimal J., Kramer M., Lockhart T., Pendergast M., Pielke R., Randerson D., Shreffler J. y Wyngaard J. (1982). The workshop on the representativeness of meteorological observations, June 1981, Boulder, Colorado. B. Am. Meteorol. Soc. 63 (7), 761-764.

Oakes M., Baxter L. y Long T.C. (2014). Evaluating the application of multipollutant exposure metrics in air pollution health studies. Environ. Int. 69, 90-99. http:// doi.org/10.1016/j.envint.2014.03.030

Piersanti A., Vitali L., Righini G., Cremona G. y Ciancarella L. (2015). Spatial representativeness of air quality monitoring stations: A grid model-based approach. Atmos. Pollut. Res. 6, 953-960. http://doi. org/10.1016/j.apr.2015.04.005

Posada J.J., Farbiarz V. y González C.A. (2011). Análisis del "pico y placa" como restricción a la circulación vehicular en Medellín-Basado en volúmenes vehiculares. Dyna 78 (165), 112121.

RE (2016). Comprehensive air quality model with extensions. Version 6.3. Guía del usuario, Ramboll Environmental. California, EUA, 286 pp.
Righini G., Cappelletti A., Ciucci A., Cremona G., Piersanti A., Vitali L. y Ciancarella L. (2014). GIS based assessment of the spatial representativeness of air quality monitoring stations using pollutant emissions data. Atmos. Environ. 97, 121-129. http://doi.org/10.1016/j. atmosenv.2014.08.015

Rosenbaum R.K., Margni M. y Jolliet O. (2007). A flexible matrix algebra framework for the multimedia multipathway modeling of emission to impacts. Environ. Int. 33, 624-634. http://doi.org/10.1016/j. envint.2007.01.004

Stevens G., Foyc B., Westd J.J y Levy J.I. (2007). Developing intake fraction estimates with limited data: Comparison of methods in Mexico City. Atmos. Environ. 41, 3672-3683. http://doi.org/10.1016/j. atmosenv.2006.12.051

Tainio M., Holnicki P., Loh M.M. y Nahorski Z. (2014). Intake fraction variability between air pollution emission sources inside an urban area. Risk. Anal. 34, 2021-2034. http://doi.org/10.1111/risa.12221

UNEP (2016). Global guidance for life cycle impact assessment indicators: Volume 1. United Nations Environment Programme. Guía. Paris, Francia. 166 pp.

USEPA (2011). Exposure factors and book, 2011 edition. United States Environmental Protection Agency. Reporte. Washington D.C., EUA, 1436 pp.

Vitali L., Morabito A., Adani M, Assennato G., Ciancarella L., Cremona G., Giua R., Pastore T., Piersanti A., Righini G., Russo F., Spagnolo S., Tanzarella A., Tinarelli G. y Zanini G. (2016). A Lagrangian modelling approach to assess the representativeness area of an industrial air quality monitoring station. Atmos. Pollut. Res. 7, 990-1003. http://doi.org/10.1016/j. apr.2016.06.002

WHO (2006). Air quality guidelines global update 2005. World Health Organization Regional Office for Europe. Directrices. Copenhague, Dinamarca, 496 pp.

WHO (2016). Ambient air pollution: a global assessment of exposure and burden of disease. World Health Organization. Informe. Ginebra, Suiza, 121 pp. 